\title{
A Comprehensive Survey on Mobility-Aware Device-to-Device Communications: Principles, Practice and Challenges
}

\author{
Muhammad Waqas, Member, IEEE, Yong Niu, Member, IEEE, Yong Li, Senior Member, IEEE, Manzoor Ahmed, \\ Depeng Jin, Member, IEEE, Sheng Chen, Fellow, IEEE, and Zhu Han, Fellow, IEEE.
}

\begin{abstract}
Device-to-device (D2D) communication proposes a new epitome in mobile networking to avail data exchange between physically proximate devices. The exploitation of D2D communication enables mobile operators to harvest short range communications for improving network performance and corroborating proximity-based services. In this article, we investigate mobility aspects of D2D communication, which are indispensable for the adoption and implementation of D2D communication technology. We present an extensive review of the state-of-theart problems and the corresponding solutions for encouraging the exploitation of mobility to assist D2D communication. Specifically, by identifying the mobility models, traces, problems, requirements, and features of different proposals, we discuss the lessons learned and summarize the advantages of mobility-aware D2D communication. We also present open problems and highlight future research directions concerning D2D communication applications in real-life scenarios. To the best of our knowledge, this is the first comprehensive survey to address mobility-aware D2D communication, which offers insight to the underlying problems and provides the potential solutions.
\end{abstract}

Index Terms-Device-to-device communication, mobility, mobile communication, mobile data traffic.

\section{INTRODUCTION}

D EVICE-to-device (D2D) communication takes the advantage of opportunistic encounters by mobile users with each other [1]. These opportunistic encounters' information between users are highly related to their movement. By exploiting users' movement, D2D enabled applications and services visualize highly opportunistic and unpredictable human mobility. Therefore, the challenges of exploiting mobility resides in the inherent complexity of users' mobility. It is mainly concerns with predicting the establishment of communication links among D2D users. For instance, two mobile users can establish a link at the time when they are in close proximity to each other. However, there are two key questions have to

M. Waqas (wa-j15@mails.tsinghua.edu.cn), Y. Li (liyong07@tsinghua.edu.cn), and D. Jin are with the Department of Electronic Engineering, Tsinghua University, China.

Y. Niu (niuy11@163.com) is with the State Key Laboratory of Rail Traffic Control and Safety, Beijing Jiaotong University, Beijing 100044, China.

M. Ahmed is with the College of Computer Science and Technology, Qingdao University, Qingdao, 266071 China. (email: manzoor.achakzai@gmail.com)

S. Chen (sqc@ecs.soton.ac.uk) is with the School of Electronics and Computer Science, University of Southampton, Southampton SO17 1BJ, U.K., and also with King Abdulaziz University, Jeddah 21589, Saudi Arabia.

Z. Han (zhan2@uh.edu) is with the Department of Electrical and Computer Engineering, University of Houston, Houston, TX 77004 USA, and also with the Department of Computer Science, University of Houston, Houston, TX 77004 USA.

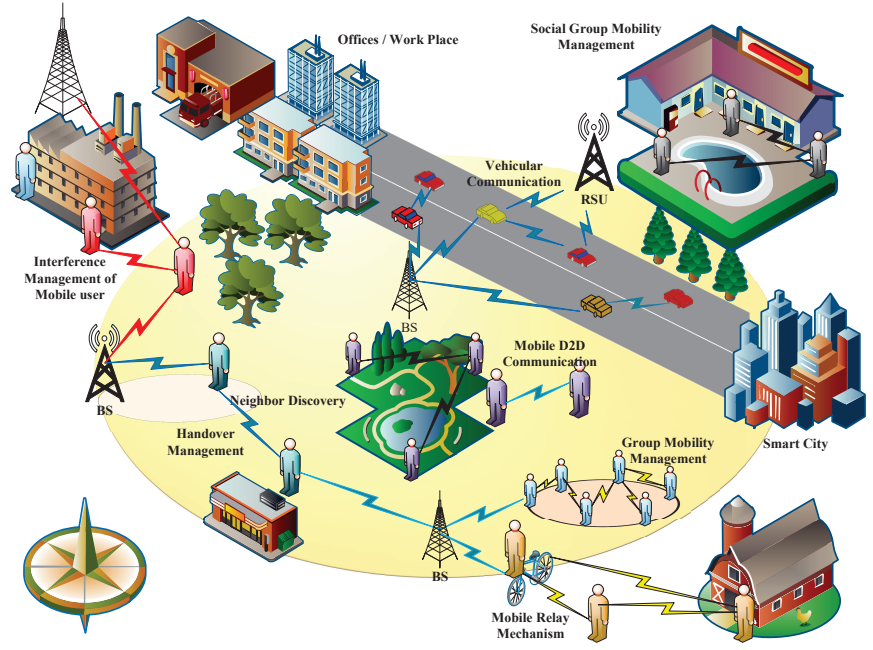

Fig. 1. Mobility assisted D2D communications.

be answered. First, can we predict the physical interactions (i.e., physical proximity) of D2D users in a mobility system? Second, when D2D users are in physical proximity to each other, can we predict whether these D2D users will stay physically close in the next time slot? Thus, mobility imposes constraints on users' mobility and/or proximity prediction. Various constraints of mobility aware D2D communications are illustrated in Fig. 1. As an example, the mobility is capable of assisting the people traveling to communicate and share common interests [2], [3]. Specifically, the information about accidents, congestion, tourist information, path finding, location, entertainment, and etc., can be shared among users, when they are in close proximity to each other in spaciotemporal manner [4]. In addition, people are naturally moving while communicating in work place, amusement parks, shopping centers, and etc. Subsequently, their movement affects the overall D2D system, such as link quality, network condition and traffic requirements [6]. Similarly, users also experience latency, extra signaling requirements and interrupted D2D links, caused by users' movements. Therefore, mobility constraints in different technical aspects of D2D communications (i.e., system capacity, energy efficiency, data transmission and etc.,) must be carefully investigated and characterized. Moreover, mobility requires careful investigation to understand the practical operational efficiency of future cellular-assisted D2D systems [7]. In contrast, modeling and investigating users' mobility, mobility models and traces of users due to mobility are needed for reliable performance analysis of D2D 
networks.

In result, users' mobility must incorporate for the native support of D2D communication into 5G system architecture. In fact, mobility affects the chances that users meet and establish a D2D connection. For instance, users' mobility in a D2D pair is a novel characteristic that related to unpredictable and dynamic effects such as, frequent channel variations due to relative user locations. Therefore, D2D mobility is expected to dramatically affect the resulting system efficiency, and its modeling must be made as accurately as possible. Moreover, mobility-related parameters (i.e., mobility models and mobility traces) determine individual D2D performance and overall D2D system performance. Focusing on different aspects of D2D communications, many research works have studied various mobility factors and mobility models using diverse mobility traces to evaluate the performance of D2D communications in real life scenarios. Researchers have also collected real-life vehicular and human mobility traces to understand the influence of the users' mobility in real life scenarios.

Although there exist several interesting surveys on D2D communications [8]-[16], which have brought considerable contributions and understanding related to D2D communications, yet the important area of mobility research is still in progress. In particular, a comprehensive and comparative study is lacking in the field of mobility-constrained or mobilityaware D2D communications. For instance, the most emerging problems in D2D communication such as interference management, caching \& offloading, energy efficiency, latency and etc. are not considered under the eye of dynamic environment. Therefore, a deep understanding of users' mobility, including human and vehicular mobility models and mobility traces, will provide vital insights on mobility-aware D2D communications. This motivates us to carry out a comprehensive survey and tutorial to study the impacts of users' mobility on the achievable performance of D2D-assisted communications.

Thus, this article surveys principles, practice and challenges of mobility-aware D2D communication. We aim to reveal deep insight for the utility of mobility on different technical aspects of $\mathrm{D} 2 \mathrm{D}$ communication and to provide detailed guidelines on

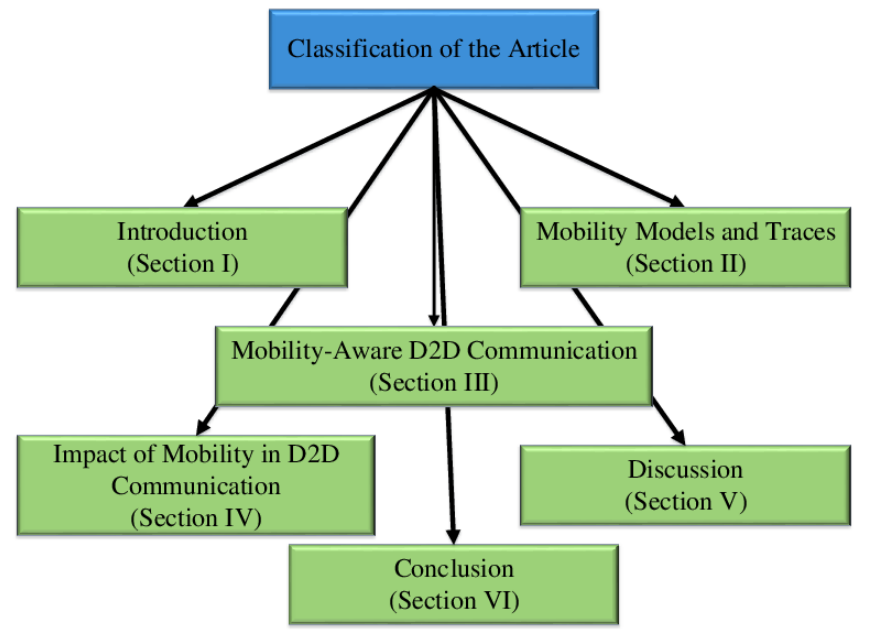

Fig. 2. Structure of the survey article. the utilization of mobility in D2D communication applications. To serve these purposes, we structure our survey paper based on mobility models and traces, technical issues of D2D communication related to mobility, and the impact of mobility on D2D communication, as summarized in Fig. 2. Building on the existing research works on mobility models and mobility traces, in Section II, first we provide a comprehensive and hierarchical classification of mobility models, adopted in D2D communication to tackle different technical issues. We then present an extensive survey on various mobility traces, collected for investigating different mobility behaviors. In Section III, we categorize distinct aspects of D2D communication due to the inherent nature of mobility. We focus on explaining these technical issues based on real-life mobility scenarios. We then investigate the impact of mobility in order to explain how mobility can assist D2D communications in Section IV. Section V summarizes the overview of mobility assisted D2D communication to discuss the key lesson learned from the existing work. It then presents challenges and future research directions for mobility-aware D2D communication. Finally, we conclude our survey in Section VI.

\section{MOBILITy MODELS AND TRACES IN D2D COMMUNICATION}

Mobility models are designed to realistically represent various real-life mobility scenarios, namely, to emulate accurately the movement patterns of various real-life applications. These mobility models allow researcher to focus on the influence of different parameters of mobility, such as speed, direction, location, contact time (CT), encounter frequency, inter-contact time (ICT), etc., on achievable performance of D2D communication. In this section, we discuss various mobility models proposed and wide-range of mobility traces collected by the research community.

\section{A. Mobility Models}

Numerous mobility models have been adopted and studied in different fields of wireless communication [49], [50]. We only consider the mobility models which are adopted by researchers in D2D communication, and we categorize these diverse mobility models according to different aspects and situations as given in Fig. 3. In this classification, we have individual mobility or group mobility models, human or social based mobility models, geographic based mobility models, user interaction based mobility models, and vehicular mobility models. We concentrate on describing how different mobility models incorporate, analyze and characterize the contact patterns of users in different scenarios. For instance, sometimes motions of users are independent of each other e.g., in pedestrian walk, while in other situations, mobilities of users are interdependent, such as in move in groups with correlated motion patterns. On the other hand, social mobility models should naturally incorporate human social interactions, and vehicular mobility models should truthfully characterize vehicular movement on road or highway in our daily life. 


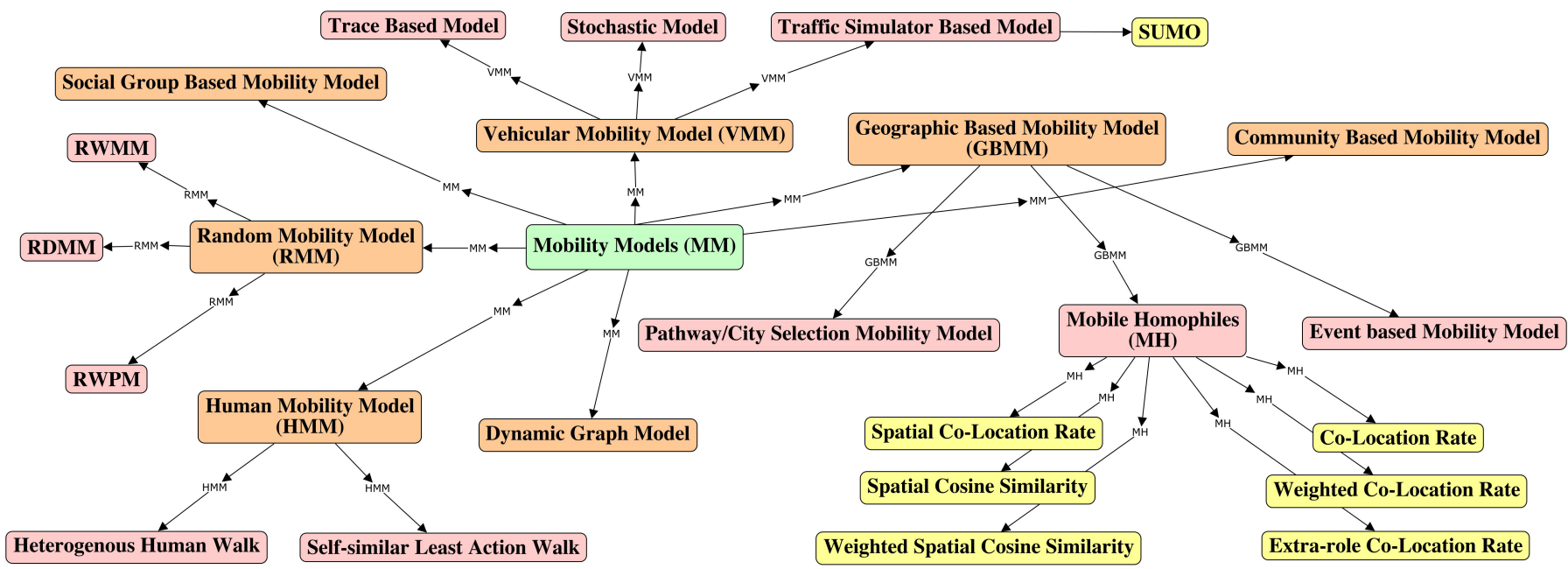

Fig. 3. Categorization of mobility models.

1) Random Mobility Model (RMM): RMM is widely adopted in D2D communication research. In this model, users are freely moving without any restriction. The speed, direction and destination are therefore random. This mobility model is memoryless, since the movements of users are independent of their previous movements. Hence, the users do not count their previous contact rates in establishing direct links. This model is further divided into random way point model, random direction model and random walk model [51]-[53].

a) Random Way Point Model (RWPM): RWPN is a frequently used mobility model due to its simplicity [54][59]. In this model, users move within a known dimensional area, and the average speed of users is distributed uniformly between minimum and maximum speeds. The velocities and directions of users are chosen independently. When D2D users come into contact, their movements stop for certain duration of time known as CT or pause-time. The maximum velocity and pause time are the two basic parameters that determine the mobility of users. Small maximum speed and long pausetime result in high stability, while fast user movement and small pause time produce highly dynamic behaviours of the users, and D2D communication may become unstable. Thus, RWPM may fail to achieve steady state, in terms of average user speed, and as a result, the speed may constantly decrease as the simulation progresses [60], [61]. However, this may not be a problem in the case of high variability in proximate users. The reason is that in this case the average number of proximate users around a specific user are continuously changing with respect to time and space in a mobile environs.

b) Random Direction Mobility Model (RDMM): It is observed that the spatial user distribution of RWPM is transformed from uniform distribution to non-uniform distribution as it reaches steady state [62], [63]. In steady state, the maximum density of nodes occurs at the center area whereas it is almost zero around the edges of the coverage area. Therefore, it is difficult to use RWPM to model the user distribution in D2D communication underlaying cellular network within the coverage area. RDMM [64] was proposed to resolve this problem of non-uniform spatial distribution of user density associated with RWPM. In RDMM, a user randomly and uni- formly selects a direction between $[0,2 \pi]$ until it reaches the edge of the area. It randomly and uniformly chooses another direction to move after reaching the edge of the coverage area and also after stopping with a pause-time. The same concept is implemented in D2D communication underlaying cellular networks [65]-[67]. However, this mobility model may not be applicable in certain D2D communication applications, such as using relay in out of coverage scenario.

c) Random Walk Mobility Model (RWMM): RWMM is originally known as Brownian motion because it emulates the unpredictable movements of the particles in the field of physics. It is adopted by the wireless communication community because it is believed that mobile users may also move in unpredictable way [68]-[73]. RWMM has certain similarity with RWPM, as user movements have randomness in the both models. However, in RWMM, user movements are purely random, and users have zero pause time when they come into contact. Therefore, the difficulty of applying RWPM to D2D communication is that D2D users do not have any information about the past state when they come in contact, because the present velocity is independent of the past velocity. This completely unpredicted randomness may not fit the real-life D2D communication scenario, because in reality the velocities at which two D2D users establish D2D link for communication may depended on their previous velocity.

2) Human Mobility Model (HMM): The aforementioned RMM is not particularly suited for human mobility, as it is unable to produce the required patterns and statistical properties to mimic real-life human mobility patterns [74]. The definition of HMM is based on physical space, temporal properties and connectivity. In physical space, proximate users usually travel in close vicinity to their home and office environments [49]. Regarding temporal properties, users are mostly return to a previous location with encounter frequency that is proportional to the rank of popular location. Thus, high success probability can be achieved to find the current location of the users by knowing the history of users' movements. The connectivity properties concern with the messages forwarded from user to user, when they come in contact. The contact duration can be measured by ICT and CT, as illustrated in Fig. 4 . 


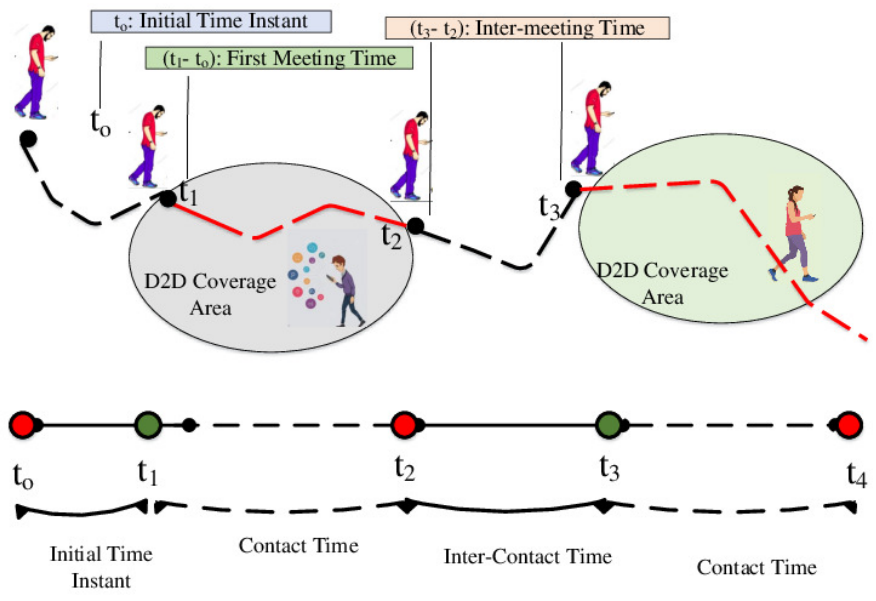

Fig. 4. Illustration of human mobility-related parameters.

Specifically, the CT measures the frequency of encounters and time length, while the ICT measures the length of the time interval from the end of the contact to the beginning of the next contact. Real-life human mobility traces are often used to find spatial and temporal conductivities of users in establishing a link between D2D users [75]-[79]. Human mobility also explores the contact duration, contact frequency, community structure, and mobile user centrality from mobility traces in real world [80]. We can further classify HMM into self-similar least action walk and heterogeneous human walk.

a) Self-similar Least Action Walk (SLAW): SLAW is one of the primary mobility models for emulating human walk behaviors [81], [82]. It has been shown that in D2D communication applications, SLAW can capture D2D users' mobility patterns [50], [74], [83]. It is particularly effective in emulating the mobility patterns of D2D users having common interest for offloading, transmitting or caching popular contents within a same geographical location such as, university campus, office or home. However, SLAW can also express the regular as well as spontaneous trip patterns present in the daily mobility of users. This is due to the reason that people typically keep a routine of visiting the same places every day such as going to an office, but at the same time, make irregular trips caused by an unexpected manner such as appointments. Thus, SLAW represents the synthetic mobility patterns i.e., regular and spontaneous.

On the other side, SLAW may not necessarily capture all the important statistical features of human mobility. There could be many other features that SLAW may not capture. One of them is temporal features of mobility. People meet because they are in the same place and also at the same "time," and also people move to a certain location at a certain time because they have to be there at that time. A hotspot may become popular only at a certain time, e.g., a restaurant area. Real mobility patterns must be a result of representing these spatial and temporal correlations. In the construction of mobility traces, SLAW chooses a set of destinations to visit randomly from a given set of hotspots, and the visits to a certain location are determined by a function of distance from the current location. While this decision process may capture some realism in spatial constraints, this does not represent temporal tendencies of human mobility. Moreover, the drawback is the exponential cut off that appears every 12 hours in the SLAW mobility model, which impacts on people's daily routines [84].

b) Heterogeneous Human Walk $(H H W)$ : The aforementioned SLAW is suitable for modeling mobility patterns of users having similar 'characteristics'. In reality, users have different characteristics and mobility behavior. For instance, some users are more 'popular' than others, as they have more friends in form of group and come into contact with other people more often. Thus, HHW aims to generate realistic and more general human mobility patterns, including the heterogeneity of human popularity. Moreover, temporal tendencies must not be conceived as an unnecessary point as in SLAW, but it is an important feature of a realistic mobility model. This holds true especially for human mobility models since temporal tendencies are what commonly prevent us from making straight line trips such as in SLAW. Therefore, it is necessary that there is indeed a need for the inclusion of temporal tendencies in order to better predict the performance of real D2D system. HHW, on the other side, have geographic constraints i.e., space and time.

Unlike SLAW, this model can describe the overlapping community/group structure which contributes to human heterogeneous popularity. A user can initiates the contact time with destination users according to their contact history to leverage mobility prediction. Since some users are more popular in community and have more chances of encountering others, they are more suitable for relaying data than non-popular users [85], [86]. Thus, users' popularity and heterogeneity in HHW is quite useful rather than the SLAW model for efficient content forwarding, data caching and offloading schemes in D2D communication [87], [88].

3) Social Group Based Mobility Model (SGMM): D2D communication and social network are correlated with each other. SGMM is founded on social network theory which allows a number of users collectively form group based on social relationships among the users [89], [90]. The grouping of users is then mapped onto a topographical space, and user movements are influenced by the strengths of social ties among users that can be changed with respect to space and time. A user in a group can implicitly reach a goal or decision point, and at this point the user decides whether to remain in the group or to move to another group, or to escape outside every group [91]. In SGMM, D2D users cooperates or communicates with each other in form of group in social network [92], [93].

4) Community based Mobility Model (CMM): The movement of mobile devices carried by human beings depends on human social behavior. Humans tend to be social and form communities in many circumstances. CMM is proposed for peer discovery scheme in social network [57], [67]. It captures the social nature of humans and is heavily dependent on the structure of relationships among people. In CMM, D2D users are divided into several groups with specific locations. The destination user is depending on the position of central user with which it has a strong social connectivity. It seems that SGMM and CMM are somehow related to each other. However these two models are different in terms of central user. In CMM, the central user of a group is static, while 
in SGMM, the central user is also moving along with other users in the group. Also CMM is different from HHW in the follow aspect. In HHW, the users' encounter frequencies are measured with the corresponding popular user based on their contact history. Hence, all the users in the group will rely on the specific popular user for data offloading, caching, and etc. However, in CMM, users form a group of community and help each other based on social phenomena without relying on a specific user's popularity. Hence, all the users in a group are dependent on each other.

5) Dynamic Graph Model (DGM): DGM enables the large scale system modeling with multiple D2D pairs and user mobility patterns, and it establishes a realistic D2D communication framework for investigating theoretical performance limits and studying the optimal system design [94]-[96]. Specifically, the dynamic transmission graph is exploited to model the spatial-temporal dynamics in terms of mobile users in the multi-hop D2D enabled cellular network that includes transmission opportunities of direct cellular transmission, multi-hop D2D connected transmission and D2D opportunistic transmission. Optimization based on DGM has been applied to the design and performance analysis of multihop D2D enabled wireless networks [97]. The optimal mobile content downloading problem in D2D communication with multiple D2D pairs and mobility patterns of users has also been formulated in [98] based on DGM. In [99], DGM is employed to investigate the fundamental problem of energy saving in D2D communication.

6) Geographic Based Mobility Model (GBMM): In GBMM, the users' movements are naturally restricted to a bounded area, such as conference hall, institution campus, office complex, shopping mall, etc. Mobility patterns are bounded, because for example, users may be guided by pathways and are congested by location. Therefore, the users' trajectories are inherently constrained by geographical closeness of the users. These trajectories are characterized by users' mobile homophilies, and are further classified into three categories which are given as follows.

a) Pathway or City Selection Mobility Model (PSMM or CSMM): In real life, D2D users often do not move randomly but move by predefined path or map. The mobility of such users is geographically constrained by the predefined map. In [100], a random graph is utilized to model the mobility patterns of D2D users based on the map of city. The D2D communication mode selection on the predefined map is characterized in [101] and [102] by deriving the mode selection map and movement based incentive, respectively.

b) Mobile Homophilies $(\mathrm{MH})$ : A natural strategy to predict the D2D links is to exploit D2D users' mobility information called mobile homophily ( $\mathrm{MH})$. $\mathrm{MH}$ defines physical quantities that capture the degrees of physically space information between mobile users. The quantities that describe the degrees of closeness in mobile users include [104].

1) Distance: The physical distance between two users' most frequent locations to make D2D links.

2) Spatial co-location rate: The probability that D2D users visit the same location without time constraint.

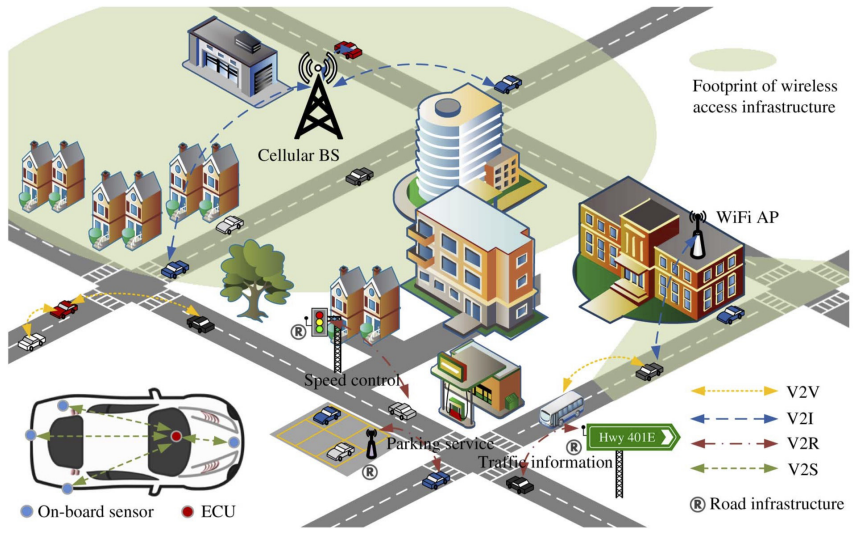

Fig. 5. Vehicular mobility infrastructure, where engine control unit is denoted by ECU.

3) Spatial cosine similarity: The probability that D2D users' trajectories are captured due to similar encounter frequencies. It is consigned by the angle between the number of encounters at the each location.

4) Weighted spatial cosine similarity: The spatial cosine similarity of each location divided or normalized by the logarithm of the population at each location. This measure promotes co-location low density areas and reprimands co-location in populated locations.

5) Co-location rate: The probability that D2D links are established at same location and time frame.

6) Weighted co-Location rate: The probability of D2D users co-locating in the same time frame, normalized by the logarithm of the population density of the co-location.

7) Extra-role co-location rate: The probability of D2D users co-locating in the same time frame at night or during weekends [106].

c) Event based Mobility Model (EMM): Another GBMM is the event based or event driven mobility model, which predicts/models the human movements caused by environmental or local events. For instance, the work [107] simulates the mobility model in a disaster recovery scenario. In an event driven situation, such as a disaster, people's movements clearly depend on their different roles in the event. For example, in a fire situation, the rescue workers are moving towards the event, while ordinary people may flee from the disaster area. EMM therefore plays a key role in the implementation of D2D communication for various disasters situations.

7) Vehicular Mobility Model (VMM): Vehicular communication has become the essential part of our intelligent transport system (ITS). Fig. 5 depicts various communication scenarios occurring in a typical vehicular mobility infrastructure or system, which include vehicular-to-sensor (V2S), V2V, vehicle-to-infrastructure (V2I), and vehicular-to-RSU (V2R) communications. VMM emulates the locomotion of vehicles along the road or highway with respect to the speed and movement, in queues and stopping at traffic signals, etc. It has wide applications, such as travel time prediction, congestion management, road safety, data dissemination, etc. High vehicular mobility imposes new challenges on the design of high-performance, low-latency and high-reliability D2D based V2V communication. D2D communication in vehicular 
environment is characterized by the inaccurate channel state information (CSI) feedback and highly dynamic spectrum management problem. Researchers have adopted different VMMs to help solving these new challenges in applying D2D communication to various vehicular scenarios [108]-[115]. Specifically, operating V2V connections in coexistence to V2I links in a D2D underlay mode is investigated in [108], in which a predictive resource allocation method and a RSU cooperative scheduling are proposed for interference control. The work [109] examines the suitability of D2D communication to safety critical Internet of vehicle application. This is achieved by qualitatively measuring the usefulness of D2D mechanism to cope with high mobility patterns. Based on VMM, the resource allocation and power control algorithm is recommended in [110] to satisfy different requirements of D2D based V2V communication and cellular communication, including intra-cell interference management between D2D and cellular links. In [111], a location dependent resource allocation scheme is proposed for automotive safety application with very strict quality of service (QoS) and reliability requirements using D2D underlay carrier. The work [112] explores efficient resource allocation strategies for D2D communication in vehicular ad hoc networks (VANETS). In this work, the authors divide the vehicles into multiple clusters on the road, and each cluster shares a single cellular user's resource.

According to the literature, we divide various VMMs into three types, i.e., trace based model, stochastic model and traffic simulator based model.

a) Trace Based Model: Many mobility traces of vehicles have been collected by different organizations on various devices like GPS, iMote, WiFi and RSUs [116]. These vehicular mobility traces can be utilized to construct trace based models for extracting the underlying mobility patterns of vehicular systems. For example, a trace based model can represent the mobility patterns of a vehicular system by different colours over a map at different time, lanes, and vehicle density.

b) Stochastic Model: In a stochastic model, the vehicle moves in a pure random motion. Such stochastic modeling can be utilized to capture the complex mobility behavior of vehicles in an ITS. The work [115] adopts stochastic modeling of VMM in the evaluation and dynamic optimization of D2D communication for ITS.

c) Traffic Simulator Based Model: Traffic simulators, like PARAMICS [117], CORSIM [118], VISSIM [119]-[121], TRANSIM [122], MONARCH [123], STRAW [124] and SUMO [125]-[127], are used to model urban traffic in order to generate vehicular mobility traces for validating a number of vehicle mobility factors. Among them, SUMO is widely used in D2D based V2V communication. This is because SUMO is a free and open traffic simulator that allows modeling of intermodal traffic systems, which includes vehicles on road, public transport and pedestrians. It can be utilized to find different tasks such as route finding, and network's visualization.

8) Summary: The goal of a mobility model is to capture the key characteristics of the underlying mobility senario and therefore to represent its mobility patterns in a realistic fashion. One way to create realistic mobility patterns would be to construct trace-based mobility models, where accurate information about the real-life mobility patterns are provided by the mobility traces. Since D2D communication have not been implemented and deployed on a wide scale, obtaining sufficient amount of real-life mobility traces is a major challenge. The synthetic models, such as RWPM, RDMM, RWMM and the stochastic model in VMM, are mathematical models develop by considering physical movements of people and vehicles. These synthetic models are not trace-driven [50]. On the other hand, there are many mobility models which are based on mobility traces. Table I summarizes various classes of mobility models discussed, where we have highlighted the main characteristics of each class, whether mobility traces are required, and whether the mobility model is connected to social network.

\section{B. Mobility Traces}

Mobility models are designed and developed to represent key parameters of mobility, such as speed, direction, location, CT, encounter frequency and ICT. These mobility parameters critically influence the achievable performance of D2D communication. However, sometimes mobility models may be ineffective to imitate the nature of mobility due to the following reasons.

1) Realistic Models: it is illustrated as whether the given mobility models are close enough to be used for network performance evaluation. This is due to the fact that some mobility models are restricted to specific scopes and could not utilized as real mobility model such as, RMM, RWPM, RDMM and RWMM.

2) Scalability: it must be ensured that whether the mobility model is able to easily incorporate an increasing number of users without influencing the diverse properties of the model. This is due to the fact that the mobility properties may effect by increasing the number of users in the system.

3) Geographical Map: some mobility models such as, CMM, DGM, GBMM and MH require geographical map for the movement of users. Without specific maps and known geographical knowledge, we will unable to imitate the mobility models.

4) Simulation: It is difficult for some mobility models to easily simulate due to complexity or computational overhead. For instance, a large of vehicles in a VMM or a large number of people in HHW may effect the true nature of these mobility models. In addition, some models are too heavy due to which they can not observe the scalability property.

In order to create a realistic mobility model that accurately emulates the mobility patterns of real-life application, it is highly effective to directly infer or derive these key mobility parameters from real-life mobility data or traces. Therefore, considerable attention has been focused on designing and collecting real-life mobility traces. In particular, some researchers utilize the traces of social network, and some exploit the traces of opportunistic networks, while others collect the mobility traces of cellular networks. Therefore, it is necessary to discuss those mobility traces that are used or can be used 
TABLE I

SUMMARY OF MOBILITY MODELS.

\begin{tabular}{|c|c|c|c|c|c|}
\hline $\begin{array}{l}\text { Mobility } \\
\text { Model }\end{array}$ & Mobility Scenario & $\begin{array}{l}\text { Trace } \\
\text { required }\end{array}$ & Heterogeneity & $\begin{array}{l}\text { Social } \\
\text { networks }\end{array}$ & Description \\
\hline RMM & Random & No & No & No & $\begin{array}{l}\text { Not consider spatial temporal properties, } \\
\text { not suitable for D2D communication. }\end{array}$ \\
\hline HMM & Random & Yes & Yes & Yes & $\begin{array}{l}\text { Based on spatial temporal properties of } \\
\text { mobility patterns. }\end{array}$ \\
\hline SGMM & Group/Community/Geographic location & Yes & Yes & Yes & $\begin{array}{l}\text { Based on spatial temporal properties of } \\
\text { mobility patterns. }\end{array}$ \\
\hline CMM & Group/Community/Geographic location & Yes & No & Yes & $\begin{array}{l}\text { Applicable for communities based on } \\
\text { geographic locations. }\end{array}$ \\
\hline DGM & Graph based model & Yes & No & - & $\begin{array}{l}\text { Applicable for multi-hop D2D connection } \\
\text { and opportunistic transmission. }\end{array}$ \\
\hline GBMM & Geographical locations & Yes & No & No & $\begin{array}{l}\text { Applicable for D2D communication in } \\
\text { restricted locations. }\end{array}$ \\
\hline VMM & ITS/Geographic locations & Yes/No & Yes & No & $\begin{array}{l}\text { Based on geographic locations, some may } \\
\text { require huge amount of vehicle traces. }\end{array}$ \\
\hline
\end{tabular}

in future for D2D communication. According to the literature of mobility traces and referring to Table II, we provide the detailed information about various mobility traces and trace collection procedures.

1) MIT Reality [128]: This data set captures communication, proximity, location and activity information from 100 MIT staff and students over the 2004-2005 academic year (9 months). This MIT data set is widely used for mining human behavior and studying properties and structure of social networks. Because this trace captures the real-life human mobility patterns, which are inherently connected with social networks, it is particularly useful for investigating D2D communication involving human mobility. For example, the work [90] utilizes this mobility trace to study social-aware D2D communication underlaying cellular networks.

2) Infocom05/06 [129]: Infocom05/06 data traces include a group of users at IEEE Infocomm conferences in 2005 and 2006 at Miami, USA and Barcelona, Spain, respectively. The data sets involve static, mobile as well as external devices. Majority of the researchers use these two data sets for proximate applications. Since the data sets measure the actual wireless networking opportunities and data transfer opportunities, they are also widely used in the field of D2D communication.

3) Cambridge [129]: This data set also includes a group of users who carry iMote devices in office and city environments. The duration of the data set is up to 12 days, and the trace records contact information and data transfer opportunities for the users at various locations, such as office, grocery stores, markets, shopping centers, around the city of Cambridge, UK. This trace has been utilized to help solving different technical problems of D2D communication due to mobility constraints as well as to exploit D2D communication based social networking [57], [146], [147].

4) Kaist/wibro [130]: Kaist/wibro data set is collected from the network traffic on the subway lines of WiBro network in Seoul, South Korea, with the speed of almost $90 \mathrm{~km} / \mathrm{h}$ with duration of approximately 1-2 minutes between two consecutive stations. This vehicular mobility trace is recorded to evaluate the QoS of voice over Internet protocol (VoIP), and it is useful for observing the performance of a vehicular system with D2D communication.
5) Intel home [131]: In this data set, connectivity and UDP/TCP throughput data are collected from a grid of six nodes placed at three different houses, 2 in USA and 1 in UK. Each node is instructed to run an experiment toward every other node in turn to assess success/loss rate, transmission rate and transmission power of proximate users. The trace also records the impact of node locations.

6) Sigcomm2009 [132]: The trace is compiled during Sigcomm 2009 at Barcelona, Spain from around 100 smart devices. Each device is initialized with the social profile that includes the information of the user's home city, country and affiliation. Each user is advised to $\log$ on to the Facebook profile, which helps the user to find friends and interest in the mobile social network. This trace presents opportunistic data transmission, encounter frequency and social profiles of proximate users in the scenario of D2D communication.

7) Rome taxi [133]: The dataset comprises the mobility traces of around 320 taxis in the central Rome, Italy, collected over 30 days. The data center collects taxis current locations, speeds, heading angles, status, etc., every $7 \mathrm{~s}$. Proximate taxi drivers can exchange the information among them for picking and dropping people according to their desirable positions and destinations. This trace is useful in investigating D2D based V2V communication.

8) Diesel bus [134]: The dataset records the data traces of 30-40 buses equipped with WiFi capabilities, which are sparsely covering almost 150 miles. It measures the intermittent connectivity between buses. Researchers exploit this vehicular mobility trace for DTN research as well as for D2D based V2V communications [38], [148]-[151].

9) Dartmouth Campus [135]: The dataset is collected for the period of 5 years at Dartmouth College, a large college campus having 190 buildings and large number of students and faculty. There are 450 APs installed with range of about 130-150 feet indoors, serving several thousands users. The data set records the mobility traces of users whose mobile devices can access wireless APs [39], [40]. The estimated movements of mobile users in between APs can be erroneous due to relatively long distance between APs. Thus, the data traces are not entirely satisfactory to explain the detailed human mobility trajectories due to an uneven granularity [152]. 
TABLE II

SUMMARY OF MOBILITY TRACES.

\begin{tabular}{|c|c|c|c|c|c|c|c|}
\hline Ref. & Year & Dataset & Size & Duration & Description & Contributor & Link \\
\hline$[128]$ & $\begin{array}{l}2004- \\
05\end{array}$ & MIT Reality & $\begin{array}{l}100 \text { smart } \\
\text { phones }\end{array}$ & 9 months & $\begin{array}{l}\text { Communication, } \\
\text { proximity, location, } \\
\text { activity information }\end{array}$ & MIT Media Laboratory & web.media.mit.edu/ nathan \\
\hline$[129]$ & 2009 & Infocom $05 / 06$ & $\begin{array}{l}98 \text { mobile } \\
\text { devices }\end{array}$ & 3 days & Office, conference & Haggle Project & crawdad.org/cambridge/haggle/ \\
\hline [129] & 2009 & Cambridge & $\begin{array}{l}8-78 \\
\text { iMotes }\end{array}$ & $\begin{array}{l}\text { Up to } 2 \\
\text { months }\end{array}$ & $\begin{array}{l}\text { Office, conference, } \\
\text { city environments }\end{array}$ & $\begin{array}{l}\text { University of Cambridge } \\
\text { Computer Laboratory }\end{array}$ & crawdad.org/cambridge/haggle/ \\
\hline$[130]$ & 2008 & Kaist/wibro & $\begin{array}{l}38 \\
\text { stations }\end{array}$ & 1 day & Vehicular network & $\begin{array}{l}\text { Division of Computer } \\
\text { Science, KAIST }\end{array}$ & crawdad.org/kaist/wibro/ \\
\hline$[131]$ & 2006 & Intel home & 6 nodes & $\begin{array}{l}\text { A few } \\
\text { days }\end{array}$ & $\begin{array}{l}\text { Home wireless } \\
\text { networks }\end{array}$ & $\begin{array}{l}\text { Intel Research, Intel } \\
\text { Corporation }\end{array}$ & crawdad.org/intel/home/ \\
\hline$[132]$ & 2012 & Sigcomm 2009 & $\begin{array}{l}100 \text { smart } \\
\text { devices }\end{array}$ & 4 days & $\begin{array}{l}\text { Opportunistic nets, } \\
\text { social profiles }\end{array}$ & $\begin{array}{l}\text { Paris Research and amp } \\
\text { Innovation Center }\end{array}$ & crawdad.org/thlab/sigcomm2009/ \\
\hline$[133]$ & 2014 & Rome taxi & 320 taxis & 30 days & Vehicular networks & - & crawdad.org/roma/taxi/ \\
\hline$[134]$ & 2008 & Diesel bus & $\begin{array}{l}30-40 \\
\text { buses }\end{array}$ & $\begin{array}{l}\text { cover } 3 \\
\text { years }\end{array}$ & Vehicular DTNs & $\begin{array}{l}\text { University of } \\
\text { Massachusetts, Amherst }\end{array}$ & crawdad.org/umass/diesel/ \\
\hline [135] & 2009 & $\begin{array}{l}\text { Dartmouth } \\
\text { campus }\end{array}$ & $\begin{array}{l}2400 \\
\text { laptops }\end{array}$ & 5 years & $\begin{array}{l}\text { Wireless network at } \\
\text { Dartmouth College }\end{array}$ & Dartmouth College & crawdad.org/dartmouth/campus/ \\
\hline$[136]$ & 2007 & $\begin{array}{l}\text { Shanghai } \\
\text { taxi }\end{array}$ & 4000 taxis & 1 month & Vehicular network. & - & {$[136]$} \\
\hline [137] & 2007 & Beijing taxi & $\begin{array}{l}27000 \\
\text { taxis }\end{array}$ & 1 month & Vehicular network. & - & {$[137]$} \\
\hline$[138]$ & 2016 & Upb/hyccups & $\begin{array}{l}72 \text { mobile } \\
\text { devices }\end{array}$ & 2 months & $\begin{array}{l}\text { Wireless contacts, } \\
\text { social connections, } \\
\text { user interests }\end{array}$ & $\begin{array}{l}\text { University Politehnica of } \\
\text { Bucharest }\end{array}$ & crawdad.org/upb/hyccups/ \\
\hline [139] & 2016 & Uoi/haggle & $\begin{array}{l}8-78 \\
\text { iMotes }\end{array}$ & $\begin{array}{l}15 \\
\text { months }\end{array}$ & $\begin{array}{l}\text { Opportunistic } \\
\text { contacts, social } \\
\text { connections, user } \\
\text { interests }\end{array}$ & University of Ioannina & crawdad.org/uoi/haggle/ \\
\hline$[140]$ & 2016 & $\begin{array}{l}\text { Oviedo/ } \\
\text { asturies-er }\end{array}$ & $\begin{array}{l}229 \text { smart } \\
\text { devices }\end{array}$ & 1 year & $\begin{array}{l}\text { Opportunistic } \\
\text { contacts }\end{array}$ & University of Oviedo & crawdad.org/oviedo/asturies-er \\
\hline [141] & 2017 & $\begin{array}{l}\text { Copelabs/ } \\
\text { usense }\end{array}$ & $\begin{array}{l}\text { smart } \\
\text { devices }\end{array}$ & 1 year & social interactions & COPELABS & crawdad.org/copelabs/usense/ \\
\hline [142] & 2016 & Kth/rss & - & - & $\begin{array}{l}\text { Sensor network, } \\
802.11 \text { ad-hoc, } \\
802.11 \text { infrastructure }\end{array}$ & $\begin{array}{l}\text { KTH - Royal Institute of } \\
\text { Technology }\end{array}$ & crawdad.org/kth/rss/ \\
\hline$[143]$ & 2015 & $\begin{array}{l}\text { Unical/ so- } \\
\text { cialblueconn }\end{array}$ & $\begin{array}{l}35 \\
\text { devices }\end{array}$ & 5 days & $\begin{array}{l}\text { Social network, } \\
\text { DTN }\end{array}$ & University of Calabria & crawdad.org/unical/socialblueconn/ \\
\hline [144] & 2013 & $\begin{array}{l}\text { Nottingham } \\
\text { mall }\end{array}$ & $\begin{array}{l}17 \text { mobile } \\
\text { devices }\end{array}$ & 6 days & Wireless contacts & $\begin{array}{l}\text { Universities of Leeds } \\
\text { and Nottingham }\end{array}$ & crawdad.org/nottingham/mall/ \\
\hline [145] & 2012 & $\begin{array}{l}\text { Tecnalia/ } \\
\text { humanet }\end{array}$ & 56 users & 6 weeks & Bluetooth & - & crawdad.org/tecnalia/humanet/ \\
\hline
\end{tabular}

10) Shanghai taxi [136]: This dataset records the real motion traces from about 4000 operational taxis carrying GPS devices in Shanghai, China, for the duration of one month. The data set contains the information of longitude and latitude coordinates of taxis' current location, time duration, speed and heading angle, and status. Although Shanghai taxi trace dataset is much larger than Rome taxi trace dataset and can be used to better investigate the mobility patterns of vehicular networks, it may still be insufficient large for the purpose of studying the large-scale vehicular system in high speed large urban environment [42], [43], [137].

11) Beijing taxi [137]: This dataset collects the mobility track logs of 27000 participating taxis in Beijing, China, for the duration of one month. This large vehicular mobility trace is widely utilized to study large-scale vehicular systems in large urban environments [29], [153]-[156].

12) Upb/Hyccups [138]: This dataset collects the mobile users' interaction information, e.g., statistical usage, activities of users, battery usage, sensor data. In addition, it also accumulates the information of users about their encounters with other users. Therefore, the mobility traces of this dataset include wireless contacts, social connections and users' interests.

13) Uoi/Haggle [139]: The traces of this dataset are derived from Cambridge traces. They embrace proximate devices and include connectivity traces that can be used for network simulation with opportunistic network environment simulator.

14) Oviedo/Asturies-er [140]: The dataset consists of the mobility and connectivity traces, which are extracted from GPS traces and collected by the regional Fire Department of Asturias, Spain. A total of 229 devices are involved in $19,462,339$ locations, where a new location is reported within an interval of approximately 30 seconds.

15) Copelabs/Usense [141]: The dataset is concerned with the social interaction of the users, and their propinquity based on wireless networking. The data are collected from the smart devices carried by people to communicate with each other in the same affiliation during their daily routines, such as at home, office, leisure activities, meeting and conference, etc.

16) Kth/rss [142]: This dataset contains the radio signal strength (RSS) data collected from a mobile robot in two environments: indoor at a KTH's office and outdoor at an abandoned steel factory in Dortmund, Germany. The position 
and orientation data of the mobile robot are collected with the aid of the robot operating system.

17) Unical/socialblueconn [143]: The dataset contains the social profiles of the participants, such as Facebook friends and self-declared interests.

18) Nottingham mall [144]: This dataset collects the Bluetooth contact traces from shop employees of a shopping mall to investigate the mobility patterns of a real-world scenario.

19) Tecnalia/humanet [145]: The dataset contains Bluetooth connectivity, proximity and mobility data, having 5 million traces with a minimum granularity of 5 seconds.

\section{Mobility-AwARE D2D COMMUNiCATION}

The mobility patterns or parameters of the underlying physical environment critically impact the achievable performance of a D2D communication system. A D2D communication technique must be 'mobility aware' in order to be effective, and researchers have carefully addressed diverse technical problems of D2D communication with the aid of various mobility models/traces. This is also the focus of this section.

\section{A. Mobility-Aware Throughput Optimization}

D2D communication typically shares resources with traditional cellular communication, which imposes serious interference. In order to mitigate interference and consequently to increase the system capacity, users' mobility behaviors must be carefully analyzed [96]. A number of research works aim to maximize the throughput by utilizing users' mobility information. For example, the work [98] optimizes the content downloading via D2D communication and the system capacity. Specifically, the throughput optimization problem is formulated by introducing the dynamic transmission graph model for large-scale networks [98]. Since conventional dynamic graph (DG) theory does not deliver the spatio-temporal locations for users, time expanded graph is proposed in [98] to enhance DG theory so that the dynamic accessing and time varying communication opportunities can be characterized. The opportunity is transient in time expanded graph to obtain the interested contents via D2D communication, and users have to wait for their preferred and interested content. The work [147] proposes the expected available duration (EAD) metric to evaluate throughput optimization by taking into account the pairwise connectivity among mobile users for their preferred and interested objects. Although, the authors of [147] show good results for their proposed scheme, this work is not suitable for large-data applications, such as video streaming and online gaming which impose strict latency and consistency requirements [157]. The separate resource block and power allocation (SOLEN) algorithm is proposed in [110] to maximize the cellular users sum rate under while satisfying the latency and consistency constraints.

Relay-aided multihop communication is an effective means to overcome poor channel quality between a mobile user and its serving BS. However, mobility causes frequent relay switching which results in increased energy consumption, potential delay and outage. The work [68] proposes a distancebased relay selection scheme. However, this scheme is not suitable for high speed applications because users' distances change rapidly. The study [158] investigates the architecture of mobile relay suitable for high speed scenarios. To improve the efficiency of the mobile relay scheme, the system can be optimized with self-optimization network (SON), power saving, measurement and system information acquisition mechanisms. In order to provide less expensive infrastructure and operating expenses for mobile relay mechanism, a virtual infrastructure topology can be considered to improve the system capacity and network coverage [159].

Some researchers address the impact of mobility not from users' perspective but from angle of dynamic network [160]. For instance, a utility function as well as contention graphbased mobility-assisted resource allocation and power control algorithms are proposed to investigate the network dynamics [56]. The authors apply a hedonic coalition formation game for mode selection and resource allocation to mitigate interference in a distributed manner, which trades off between the achievable rate and the costs. The power control problem is also formulated to maximize the sum rate of the cellular links in the coalition formation. The coalition formation is suitable for fixed-location or slow moving mobile users. Therefore, location dependent resource allocation is proposed in [111]. Specifically, the authors propose a heuristic location dependent resource allocation scheme (LDRAS). to reduce the signaling overhead and interference level. However, the work [111] only considers a single cell scenario with limited users. Botsov et al. [161] extend this work to multi-cell deployments. The authors utilize LDRAS to satisfy the requirements of cooperative intelligent traffic system safety services, while reducing the signal overhead and interference within the network.

In future-generation cellular networks, D2D users share resources with cellular users. Interference management, resource allocation and mode selection are all influenced by the underlying mobility patterns of mobile users. Table III lists various mobility-aware techniques for throughput optimization and system capacity enhancement discussed in this subsection. How to utilize mobility information to assign resources optimally in highly dynamic environments is still an open issue.

\section{B. Mobility-Aware D2D Data Offloading/Caching}

Mobile data offloading and caching via D2D communication will play a key role in meeting the explosive increase of mobile data traffic. Traffic offloading in cellular networks includes local voice services, multimedia content sharing, gaming group completion context aware applications, public safety, etc. From network operator's perspective, networks can offload mobile traffic to users via D2D communication to avoid network congestion and to optimize the performance of voice and data services [162], [163]. However, the mobility constraints of D2D users impose serious in mobile data offloading and coaching. An effective D2D offloading and caching scheme must carefully consider the mobility properties of the underlying mobile environment, i.e., it must be mobility aware.

In this regard, some optimization techniques are proposed to investigate the mobility affect on data offloading and caching. For instance, Wang et al. [87] study the problem 
TABLE III

MOBILITY-AWARE THROUGHPUT OPTIMIZATION.

\begin{tabular}{|c|c|c|c|c|c|c|c|c|}
\hline Ref. & Year & Target & Network & Model/Trace & Technique & Mobility speed & Devices & Type \\
\hline$\overline{[56]}$ & 2015 & $\overline{\mathrm{D} 2 \mathrm{D}}$ & Cellular & RMM/- & DG theory & Human & - & - \\
\hline [96] & 2016 & D2D & Bluetooth & SLAW/Kaist & DG theory & Human/Vehicular & varying/92 & GPS receivers \\
\hline [98] & 2014 & D2D & Bluetooth & SLAW/Infocom05 Kaist & DG theory & Human/Vehicular & varying/41/92 & iMotes \\
\hline [110] & 2016 & V2V & Cellular & VMM/- & SOLEN & Vehicular & 50 & Vehicles \\
\hline [111] & 2014 & V2V & Cellular & VMM/- & LDRAS & Vehicular & 80 & Vehicles \\
\hline [147] & 2016 & D2D & Bluetooth & -/Intel Infocom Cambridge & EAD metric & Human & $8 / 12 / 41$ & iMotes \\
\hline$[158]$ & 2013 & - & WiFi & GBMM/- & SON, etc. & High-speed vehicular & - & Vehicles \\
\hline$[161]$ & 2015 & V2V & ITS & SUMO/- & LDRAS & Vehicular & 320 & Vehicles \\
\hline
\end{tabular}

TABLE IV

MOBILITY-AWARE D2D DATA OFFLOADING/CACHING.

\begin{tabular}{|l|l|l|l|l|l|l|l|l|l|}
\hline Ref. & Year & Target & Network & Model/Trace & Technique & Mobility speed & Devices & Contact & Type \\
\hline \hline$[55]$ & 2015 & D2D & Cellular & RWPM/- & IADOA & $20 \mathrm{~m} / \mathrm{s}$ & 20 & - \\
\hline$[87]$ & 2014 & D2D & Bluetooth & HMM/Infocomm05 & Stochastic & Human & up to 100 & 3 days & Smart devices \\
\hline$[88]$ & 2014 & D2D & Cellular & HHM/- & Social ties & - & 32 & - & Smart devices \\
\hline$[92]$ & 2016 & D2D & WiFi & GBMM/- & PETOS & - & 6 & - & Smart devices \\
\hline$[93]$ & 2015 & D2D & Cellular & GBMM/- & Game theory & - & 400 & - & Smart devices \\
\hline$[102]$ & 2016 & D2D & WiFi & PSMM/Sigcomm09 & Stochastic & Human & 76 & 96 hours & - \\
\hline$[146]$ & 2017 & D2D & Bluetooth & GBMM/Infocom06 & Game theory & Human & 98 & 3 days & iMote \\
\hline$[164]$ & 2013 & D2D & Cellular & /Sensor Mote $[172]$ & IBP & Human & 27 & 79 days & T-Mote \\
\hline$[165]$ & 2015 & D2D & Cellular & MH/Infoc. MIT SUVnet $[173]$ & TASA & Human & $41-4311$ & $4-246$ days & iMote \\
\hline$[167]$ & 2016 & D2D & Bluetooth & MH/Infocom06 MIT reality & sNDN & Human & $78-97$ & $4-246$ days & iMote \\
\hline
\end{tabular}

of mobility assisted opportunistic offloading problem with the help of statistical properties of users to offload the computation tasks among users. The problem is further investigated for data offloading and caching by considering different mobility patterns and content sizes of data files in [55]. Specifically, the authors of [55] propose the distributed infrastructure-assisted data offloading algorithm (IADOA) by utilizing the contacts information between mobile nodes, provided by the BS. Huang et al. [92] propose the power efficient traffic offloading scheme (PETOS) to provide the group location-based service (LBS) and group-based caching, in order to reduce cellular traffic and power consumption in D2D communication. A group of users travel outdoors together to form mobile coverage network by utilizing smart phones to download and share LBS data to their neighboring nodes. PETOS takes into account user mobility by providing dynamic group management protocol that allows the users for one hop dynamic network coverage. The movement based incentive mechanism is also used in relay selection and payment determination [102]. However, the scheme of [102] is limited to the senario where potential relays are mobile, while D2D users are static. The proposed mechanism utilizes the mobility of relays to improve the performance of traffic offloading. To motivate and control mobile nodes to move from one place to other, the mobile operators can provide some incentives to these potential relays for their cost [103].

Mobile Internet contributes to most of the rapidly-increasing mobile data traffic Users are mostly attracted to and requested frequently popular contents, such as viral videos and new online gaming. Downloading the same popular contents by users through cellular network results in huge amount of multiple duplicate transmissions. In order to reduce the burden on cellular network, Zhang et al. [164] propose a social network assisted approach for D2D communication. Although the connectivity among users can be intermittent, social relations in real world tend to stable with respect to time. By exploiting the stable social tiers, the authors of [164] use the Indian buffet process (IBP) to model the content dissemination links and develop an online traffic offloading algorithm. Another socialaware mobile data offloading scheme is presented in [88], by leveraging the notions of users' pattern prediction, storage and social networking. The work [88] shows that the peak traffic demands can be significantly reduced by proactively serving predictable user demands via caching.

Game theories have also been to investigate mobilityaware caching and offloading. For example, Wang et al. [93] propose a network formation game to capture the dynamic characteristics of the users. In the network formation game, the users decide their D2D sharing strategies based on the historical records of mobility patterns and cost ratios between cellular and D2D transmissions. The work [146] classifies users into multicast and core types with the consideration of users' mobility and social characteristics, such as user stay probability and familiarity. Stackelberg game based pricing mechanism is then proposed to motivate the core users to distribute the video data offloading, in order to alleviate heavy BS traffic load for video services. A Tag-assisted social-aware (TASA) opportunistic sharing scheme is proposed in [165], [166] for users to share the content through local opportunistic connectivity in mobile social networks. A social-aware named data networking (sNDN) framework is exploited in [167] to form friendship group in a close proximity based on encounter frequencies for content retrieval via D2D communication.

The users' mobility parameters, including contact time, contact frequency and offloading probability, can be utilized for D2D assisted wireless caching systems [168], [169]. The joint mobility aware and small cell BS density caching placement schemes are proposed to bring the impact of user mobility and small cell BS distribution on the caching placement. 
It is pointed out that mobility can effect positively on the computation offloading in order to improve the performance in term of energy cost. However, an incentive mechanism is considered for a dynamic network to differentiate user's QoE and the heterogeneity of mobile users in terms of caching and computational capabilities. Thus, Shabani et al. [163] investigated the effect of mobility on D2D coded caching architecture. The authors compared the work with the static environment and concluded that the coded multi-casting gain and the spatial reuse gain can be achieved simultaneously, by leveraging mobility in a D2D coded caching network. However, the D2D users need to discover the close proximity relay node according to the location and channel conditions for offloading the data. The dynamic environment is beneficial for caching in a heterogeneous network to minimize the system cost in terms of energy and bandwidth wastage [170]. It took the advantages of the storage capabilities of users in the system. The storage files are accessed by other users in the system through D2D communication. This minimizes the average system cost by reducing congestion at the backhaul link in terms of energy and bandwidth wastage, and requires less computational resources.

In summary, the randomness of users' mobility contributes potentially for caching more data in the network than deterministic users. However, the node mobility affects the link quality between D2D pair due to node location over time. The connection between D2D pair can be lost or degraded due to the displacement of one of the two nodes in D2D pair, and the transmission of large files may not be successful due to mobility constraint. The problem is introduced in [171], where success probability is considered to establish D2D link. The caching transmission is initiated, when the transmitter has the desired content cached, and their location or position remained constant for certain duration of time. This achieves a satisfactory QoS for a user to keep its position for an exponential period of time before changing the position from the coverage of receiver. The mobility aware analysis captures the information of the local vicinity of users at the exact location. In order to give quick view of the overall discussion, we summarized the mobility parameters for the state-of-the-art problems in Table IV.

\section{Mobility-Aware D2D Energy Efficiency}

It is worth important to improve the energy efficiency by reducing the physical communication distance between D2D pairs. However, it is crucial to address the problems in current communication practices that lead to excessive energy waste at the node terminal and even at the infrastructure side [114]. The performance of the energy consumption is investigated with varying network factors i.e., flow delay, buffer size and bandwidth [99]. It is necessary to reduce the energy consumption for data transmission by any possible way of transmission from BS to the receivers under the human mobility constraints. Likewise, Wu et al. [180] raised the problem of uplink resource sharing in dynamic D2D communication environment. The authors construct the energy efficiency model for different sharing modes, QoS requirements, and spectrum utilization of each user. To cope with the problem, they proposed an NT-coalition formation game with the characteristic function in terms of energy gain and the costs in terms of mutual interference. Similarly, Solar et al. [181] proposed a test bed that implements link-aware opportunistic D2D transmission. Therefore, the work is extended by $\mathrm{Wu}$ et al. [175] to investigate the random mobility process, and mobility influences on Energy Efficiency (EE) and Bandwidth Efficiency (BE). The authors proposed an EE-BE aware scheduling scheme with a mobile relay selection strategy, rate allocation and routing, while investigated the relationship between energy efficiency and bandwidth efficiency. Although, the proposed scheme improved the efficiency, reliability, QoS and performance of D2D communication by reducing energy consumption, yet the real data strategies for mobility patterns and models are still a missing section.

To capture the mobility preferences and similarity for mobile encountering and prediction, the time space matrix (TSM) is introduced [176]. The mobility preferences and similarities aim to utilize the devices' mobility to create encounter opportunities. In addition, the authors in [176] further investigated the encounter frequencies between users. The approach aims to utilize the device to reduce the transmission distance, and consequently, reduce the energy consumption. Another benefit of encounter frequencies is to increase the message delivery probability at a cost of energy consumption. However, there is a strong trade-off shown in [179] between energy consumption and encounter probability. The proposed autonomic scheme is consisted of two phases. The first phase is to determine the energy cost, and the probabilistic nodes decide either to stay static or move in the second phase. It is observed that the significant energy conservation occurred due to trade-off between encounter probability and energy consumption under mobility constraint.

As the users' movement is arbitrary, it is difficult to determine mobility speed and pattern. On the other hand, mobile users are operated through batteries having limited energy capacity, hence it is a dire need of energy constrained based mobility control techniques. Energy is consumed due to transmission and reception of data contents, and nodes' mobility. In [182], the authors modeled a message dissemination process under the consideration of variable velocity of nodes by the deployment of continuous-time Markov model for data dissemination. However, most of the present solutions focus on probing inter-contact intervals, and it is assumed that contact rate follows exponential distribution. Many researchers have negated this fact and proved with experiments that contact rate follow power law distribution. On the basis of power law distributed contacts, Zhang et al. [177] proposed adaptive wakeup scheduling. The approach focuses on node wakeup during contact probability and sleep in case of low contact possibilities. In addition, Bista et al. [178] had performed comparison among Epidemic, PRoPHET and Spray-and-wait protocols under RWM, RWPM and shortest path map-based movement mobility models for evaluating utilization of resources and mainly energy consumption. The authors demonstrated that energy conservation and reduction in overhead is provided by Epidemic and PRoPHET Protocols 
TABLE V

MobiLITy AWARE D2D For ENERGY EFFICIENCY.

\begin{tabular}{|c|c|c|c|c|c|c|c|c|c|c|c|}
\hline Ref & Year & Target & Network & Model & Trace & Technique & Mobility & Devices & Contact & Type & Speed \\
\hline [99] & 2016 & D2D & Cellular & SLAW & KAIST & Network opt. & Human & 50 & - & GPS RXs & $1 \mathrm{~m} / \mathrm{s}$ \\
\hline$[114]$ & 2016 & $\mathrm{~V} 2 \mathrm{~V}$ & Cellular & SUMO & - & - & Vehicular & 20 & - & vehicles & $14 \mathrm{~m} / \mathrm{s}$ \\
\hline [175] & 2014 & D2D & Cellular & RWM & - & - & - & - & - & - & - \\
\hline$[176]$ & 2016 & D2D & Bluetooth & - & MIT/Dart. & TSM & Human & $1350-1500$ & 4 Weeks & - & - \\
\hline$[177]$ & 2014 & D2D & Bluetooth & - & Intel/Info./Cam. & Adaptive scheme & Human & $128-4724$ & - & iMote & - \\
\hline$[178]$ & 2016 & DTN & WiFi & RWP & - & - & - & $100-600$ & - & Smartphones & $1.5 \mathrm{~m} / \mathrm{s}$ \\
\hline [179] & 2016 & DTN & - & RWP & - & Game Theory & - & - & - & - & $1 \mathrm{~m} / \mathrm{s}$ \\
\hline$[180]$ & 2014 & D2D & Cellular & RWP & - & - & - & - & - & - & - \\
\hline [182] & 2011 & DTN & Cellular & - & - & $\begin{array}{l}\text { Epidemic } \\
\text { Routing }\end{array}$ & - & - & - & - & Variable \\
\hline
\end{tabular}

in RWM, whereas delivery probability is enhanced in RWPM.

From the above discussion, it is concluded that mobility of users also effect the energy consumption of the users' devices. We can see from Table $\mathrm{V}$ that different authors utilizes different mobility models, traces and mobility parameters in order to achieve better energy efficiency in a dynamic environment. However, the speed of users in the state-of-theart research is considered very low. The energy consumption in high speed scenario is not investigated in the current research.

\section{Mobility Aware D2D Latency Investigation}

It might not be feasible or reliable to compromise the latency between end users due to the mobility of users. For example, the latency might be increased due to controlling thousands of devices to exchange information with each other. In addition, the signaling overhead further affects the information delivery between end user due to mobile environment. The latency could be increased by exchanging the control signal that makes D2D communications unreasonable, when several BSs are also involved to make the handovers. To enable minimum latency data transmission between end users is one of the crucial advantages expected from D2D communications. The latency in D2D communication can occur due to fast locomotion of the users within the same cell, handovers (HOs) between different cells, random mobility impacts, high data transmission in specific time duration, and in terms of $\mathrm{V} 2 \mathrm{~V}$ [108]. D2D communication HOs problems occur due to legacy of LTE system that cannot support D2D HOs. Therefore, when D2D users perform LTE HOs, some several drawbacks might be emerged such as latency, extra signaling exchange and interrupted D2D links.

Researchers investigated the variety of these scenarios, and proposed different techniques to overcome the problem of latency in D2D communication. For instance, Yilmaz et al. [183] presented the problem of latency due to HOs. The authors proposed mobility management solution and predicted achievements for D2D communication. However, the mobility of D2D communication is considered as a HO, while it lack of mobility patterns and real environment scenarios. In the line of this, Barua et al. [188] further over viewed the mobility management system for LTE-A technology, and proposed a model and an algorithm for D2D communication HOs to lower the signaling overhead, lower delay and uninterrupted D2D communication. The $\mathrm{HO}$ problem is further investigated by
Orsino et al. [54] to enhance the QoS of mobile users at the time of its HO in LTE system. However, the proposed scheme enables the users to temporarily connect to a suitable relay via D2D link during HO. The HOs and mobility of users are specified on the current LTE-A HO procedures, and hence it cannot support the proximity services continuity requirements. It is considered that continuous HOs are expected when the proximity users move across the BS boundary. Thence, an efficient D2D $\mathrm{HO}$ mechanism is essential to meet the requirements of proximity users. Among many, one of the solution is proposed in [184]. In the article, Joint Handover Procedure (JHP) and Half Handover Procedure (HHP) are studied. In the JHP, the network considered all the proximity based users in the ongoing D2D communication due to close proximity. While in HHP, one of the users in the proximity can be handed over to the targeted BS, and the other one will still the source of BS. However, this causes a huge controlling signaling burden, interference issues and complex mobility patterns recognition over BS. Therefore, Lei et al. [115] captured stochastic nature of traffic arrivals and complex mobility behavior for dynamic optimization in terms of high reliability and low latency.

Most of the researchers have considered homogeneity in terms of users' mobility in the above mentioned works. Nevertheless, this is not true because the real traces have depicted that mobility patterns are heterogeneous. The heterogeneity in contact rates poses limitation in the performance of communication, and in result a transient communication links occurs due to the nodes' mobility. The heterogeneity problem is taken by Li et al. [186], [189] into consideration. The authors leveraged heterogeneity in delivery costs and contact rates during selection of relay nodes for achieving a reduction in delivery cost while maintaining required delivery probability of message. Optimal heterogeneous relaying scheme (OHRS) is formulated in order to select optimal relaying nodes to reduce the latency. The outcomes demonstrated that proposed approach is efficient in achieving a high delivery probability. The latency issue can also be occurred for data delivery by finding proximate users in D2D communication. The mobile application discovery protocol in a cellular environment must be efficient in radio resource usage, energy efficiency for battery life, and are able to discover as many devices as possible. Keeping the mobility issue, Zhang et al. [57] proposed a social network characteristic peer discovery scheme for D2D communications. It is concluded that the peer discovery 
TABLE VI

MOBILITY AWARE D2D FOR LATENCY.

\begin{tabular}{|c|c|c|c|c|c|c|c|c|c|c|}
\hline Ref & Year & Target & Model & Trace & Technique & Mobility & Devices & Contact & Type & Speed \\
\hline $\begin{array}{l}\text { [54] } \\
\end{array}$ & 2015 & $\mathrm{D} 2 \mathrm{D}$ & RWPM & - & D2D HOs & Vehicular & 120 & - & Vehicles & $10-100 \mathrm{~km} / \mathrm{h}$ \\
\hline$[108$ & 2015 & $\mathrm{~V} 2 \mathrm{~V}$ & VMM & - & Stochastic & Vehicular & - & - & Vehicles & - \\
\hline$[186$ & 2013 & DTN & - & Info./MIT/Shng & OHRS & Human & $41-2100$ & 3-30 (days) & - & - \\
\hline$[189$ & 2011 & DTN & - & Info./MIT/Shng & OHRS & Human & $41-2100$ & 3-30 (days) & - & - \\
\hline [57] & 2015 & $\mathrm{D} 2 \mathrm{D}$ & RWPM & Int./Info. & Social ties & Human & $9 / 78$ & $3 / 4$ (days) & iMote & - \\
\hline
\end{tabular}

ratio significantly increases with social grouping and social centrality, and improved the system performance in terms of data delivery ratio and reducing the average delivery delay. Furthermore, the authors in [67], also proposed a short discovery code, which contains compressed information of mobile applications in a device. This allows the users to find out the desirable applications in the other device while consuming small amount of radio resources. The authors considered RDMM for discovering mobile application in cellular D2D communication, to analyze the performance of the proposed discovery protocol. In [187], Zayani et al. had proposed a technique of temporal link prediction (TLP) to find similarity between a pair of user, and for the prediction of future connectivity. For this purpose, the authors have devised tensorbased link prediction algorithm for the prediction of temporal links.

It is investigated that very few works are done in the latency of D2D communication under mobility consideration as depicted in Table VI. However, mobility brings a positive role to reduce latency in D2D communication. Therefore, it is profound importance to investigate the mobility parameters and mobility behavior of users in order to reduce latency in D2D communication for better QoS and QoE. Also from the above discussion, it is pointed out that D2D communication is one of the important element for the reduction of latency during HOs.

\section{E. Mobility-Aware D2D Content Dissemination}

It is envisioned that D2D communication scenarios will flourish further that would include not only about emergency, public safety circumstances and $\mathrm{V} 2 \mathrm{~V}$ improved traffic safety situations, but also cover commercially available pre-standard products to facilitate the social-networking and peer-to-peer connections that considered out of communication coverage or in case of congestion. In addition, D2D communication is considered economical and energy efficient application when sharing or disseminating specific contents between proximity users. However, the data dissemination will become a challenging part, when all the users are in dynamic environment. Particularly, the problem of multi-copy data dissemination with probabilistic delay constraint in opportunistic D2D communication is more challenging problem [71]. The multi-copy content dissemination can be reduced to a single content dissemination to figure out the effect of mobility in D2D communication [196], [197]. The mobility factors comprise of user interaction depending on time and location. The authors investigated the problem of content transmission and resource allocation by exploiting the contact patterns of D2D users with the help of statistical properties.

In mobile social network (MSN), a wide range of user generated social media contents are propagated between users through social connectivity. A community based approach is one of the method that is presented by leveraging social encounters into the context for establishing, and ensuring the success of D2D transmission [83]. The content transmission through social network has significantly challenged the tradition data transmission paradigm due to limited bandwidth and storage capacities. For this reason, Wang et al. [190] observed that the mobility patterns and social content transmission affected the replication of the content transmission, users' movements across the region, and the discovery of neighboring peers. They utilized the distributed algorithm for historical, local and partial information of the users to solve the replication scheduling. Furthermore, Kang et al. [198], [199] considered a mobile content delivery network ( $\mathrm{mCDN}$ ) on the assumption that mobile CDN are randomly distributed by a Poisson point process. In $\mathrm{mCDN}$, special mobile devices are designated as caching serves that provide mobile stations with popular contents on demand through D2D communication links. The authors present optimum dual solution searching algorithm (ODSA) to determine the probability of storing the individual content in each server that minimizes the failure rate. In vehicular networks, RSUs are deployed along road sides enabling V2I communications but there is an explosive increase of vehicular traffics. Therefore, an improvement is required in designing RSU-aided vehicular delay tolerant networks (VDTNs). Therefore, the works in [193], [194] investigated the contact-aware mobile data replication for RSU-aided VDTNs for data dissemination.

It is also essential analyze the impact of imperfect cooperation of nodes on message replication that is crucial for multicopy forwarding protocols [192], [195]. The proposed replication mode selection procedure as a finite-horizon Continuous Time Markov Decision Process (CTMDP) with restricted decision epochs. A static spraying policy is introduced that permits source-destination space-time paths. Spray and Wait protocol are traditionally used in DTNs, which predefines maximum allowable replication of message. But there is a problem of restricting maximum number of replications as 
TABLE VII

Mobility AwARE D2D CONTENT DisSEMinATION.

\begin{tabular}{|l||l||l||l||l||l||l||l||l||l||l|}
\hline Ref & Year & Target & Model & Trace & Technique & Mobility & Devices & Contact & Type & Speed \\
\hline \hline$[71]$ & 2016 & D2D & RWMM & DieselNet & Stochastic & Vehicular & $100 / 33$ & - & Vehicles \\
\hline$[83]$ & 2017 & D2D & SLAW & Groups-NET & Stochastic. & Human & 400 & - & 3 days & - \\
\hline$[190]$ & 2017 & D2D & GBMM & Tencent & Stochastic & Human & 1.2 Millions & 10 days & Human & - \\
\hline$[191]$ & 2011 & V2V & RWP & Shanghai & Homophily & Vehicular & 2100 & 1 Month & Taxi cabs & $0.6-1.4$ m/s \\
\hline$[192]$ & 2012 & D2D & SLAW & MIT/Info. & Spray \& Wait & Human & $92 / 41$ & $270 / 3$ days & - & - \\
\hline$[193]$ & 2017 & V2V & VMM & French data & - & Vehicular & - & - & - & Vehicles \\
\hline$[194]$ & 2016 & V2V & VMM & China & - & Vehicular & $27000-2019$ & 1 month & Vehicles & - \\
\hline$[195]$ & 2017 & DTN & - & Intel/Info. & CTMDP & Human & $8 / 41$ & - & iMote & - \\
\hline
\end{tabular}

it may result in an inappropriate replication. This problem is addressed by Henmi et al. [200] through an improvement in Spray and Wait protocol by using forwarding token (FT) settings. In the proposed mechanism, node mobility is set as an indicator where a node containing message will replicate only to those nodes having higher mobility then its own.

colorredThe data density is measured for the amount of data disseminated by a particular device over area. The fairness among devices indicates an effective dissemination of data among a considered area. This is because when we consider different users' mobility, the users with high mobility can cover a large area as they move through space, while users with low mobility stay in a similar location. The mobility parameters of the existing literature are as summarized in Table VII.

\section{IMPACT OF MOBILITY IN D2D COMMUNiCATION}

The 3GPP open a gateway for a rich association for mobile devices in a physically $5 \mathrm{G}$ proximity services [202]. It provides a plethora of proximity services such as, device discovery, high data transmission, low delivery delays and traffic offloading. However, the main issue is that the interactions are not static, but are stochastic by nature. Thus, it becomes highly opportunistic contacts due to the prospective mobility of all interacted users' devices. Proximate based D2D communication can facilitates an efficient link continuity with relevant proximal devices in our daily life for data sharing, forwarding and offloading [203], [204]. In contrast, the link establishment between users can be in a way that one or both users can be a mobile. In addition, it is also possible that connected users can move in any or same direction; serve in same location while promising their link continuity. Therefore, mobility of users bring various important facets such as, increased outage probability, call blocking, data failure transmission, offloading failure transmission and handovers failure mechanisms. Moreover, it is also important to bring the system wide performance on the frequent response and opportunistic contacts to the realistic users' movement.

\section{A. Impact of Users' Movement}

Researchers are trying to find out the impact of mobile users on the overall system and users performance. However, users' interactions with each other are strongly affected by the patterns of users in terms of time and space [74], [104], [175]. As discussed in the aforementioned state of the art, recent studies have shown some theoretical and experimental results to show the useful and interesting outcomes for modeling the realistic users' movements. It is because, we must envision the users' movement in our next generation wireless networks. Indeed, the users are perpetually moving, while communicating between home, office, attending leisure events, visiting shopping promenades and malls, and meeting friends, family and colleagues at different timings and geographical locations [5]. In this respect, most of these activities are concentrated in metropolitan areas. Since the urbanization is anticipated to integrate more than $80 \%$ of the developed world's population by 2025 [105]. On the other hand, Someone who travels regularly from home in a suburb to work in a city (known as commuters), his/her traffic from countryside is rising because most of the workplaces are in and around metropolises. The European Environment Agency reported in 2013 that typical commutation times within larger metropolises are likely to exceed one hour per trip. However, commuters from countryside have to suffer even longer trip duration. Furthermore, to make the most of this time spent in public and private transportation, people use their mobile devices, such as smart-phones and tablets, for entertainment (watching video clips, reading news and e-books, listening to music and audio books), shopping in online stores, preparing work, scheduling appointments, socializing on web platforms, and so on. All such services require internet access, in some cases just for a few bytes while in other cases for entire data streaming, and thus depend on wireless connectivity.

In this regard, the researchers are collected different statistical traces by simply observe or use communication devices to collect mobility traces, and implemented these traces to study practical and real life D2D communication. These mobility traces can reveal the definite and repetitive patterns of human or vehicles movement. Thus, it brings an advantage to make the connection among users in time and space domain [128][135], [137]-[145].

\section{B. Role of Mobility Models}

Mobility model incorporates the spatio-temporal properties of users. It includes the distribution of the contact duration, and the distribution of the time between two consecutive contacts between users. It is also necessary to capture the human mobility traces in order to observe the users' pattern in the implementation of exact mobility model. The knowledge of human mobility behaviors is quite philosophical for the investigation of relative locations of user mobility. Therefore, 
it is well worth that the patterns/trajectories of each individual must be measured to find the exact phenomena of mobility models. In fact, $93 \%$ potential predictability is found in user mobility patterns to achieve the exact mobility models [208].

However, it is figured out that the RWMs are independent with past state, and the direction and velocity are fully stochastic in nature [209]. RWP is then considered for capturing the movement of mobile users by adding the pause time into RWMs [72]. According to [60], this model runs out to allow a steady state in which the average users' velocity systematically decrement over time. Hence, it may not be used directly for mobility patterns to capture the real human scenarios. Random way point, random direction and random walk mobility models are not suitable for D2D communication, because they do not contemplate the true nature of mobility as observed in real life scenarios. Other mobility models such as GBMM, EMM, MH, PSMM have an undesired side effects that occur since it restrict the mobile users in a certain communication range. On the other side, it is beneficial for the a certain users, moving in the same time duration and space.

Several other mobility models have been proposed and widely applied in the current research works as mentioned in Section II. However, these models are in general characterized by random movement processes due to the lack of users trajectories from real-life, and they can provide tractable analysis for D2D communication. However, it has been found that the complete randomness of mobility pattern in such mobility models can cause unrealistic moving behaviors. These unrealistic moving behaviors can lead to biased or even misleading results for D2D communication. Therefore, further investigation is necessary to adopt an exact mobility model in the area of D2D communication.

\section{Impact of Mobility on $Q o S \& Q o E$}

Mobility can affect the performance, enabled applications and services envision due to highly opportunistic device contacts. In results, it has a huge influence on system capacity, data offloading, users' QoS, users' QoE and transmission opportunities [206]. It is believed by the operators and providers that the primary challenges in D2D communication are the users' QoE and QoS. As, D2D communication normally operates in cellular mode or D2D node for proximate users to communicate each other. The users' QoS and QoE can be greatly affected by the variation of channel due to users mobility. Since the variation in the CSI of D2D links is caused by users' movement, therefore D2D links must be constantly monitored for the continuation of communication. It is important due to the fact, that the network should make mode switching between D2D link and cellular link to provide significant QoS and QoE to the users [65], [101]. It is also intriguing believe that the delivery delay is inversely proportional to the average user velocity i.e., by decreasing the user velocity, the delivery delay increases and vice versa. Therefore, mobility can increase the system capacity by decreasing the delivery delay, which in results provides a good QoS and QoE towards users [159], [164], [207].

\section{Impact of Mobility in D2D mode Selection}

The role of mobility using stochastic geometry is also applicable in D2D mode selection for both absolute and relative movements of dynamic users [58]. The users' mode transition time indicates the users' position that changes frequently with respect to time. On the other side, the users' residence time is an expected duration of users to resides in the range of D2D communication. Data transmission via D2D communication is a reliable method which does not rely on infrastructure. Thus, it is necessary to improve the data transmission using D2D transmission by a mode selection procedure according to the users' movements. However, users' mobility may be high or low depending on the velocity of the users. A mode selection function is a unique feature to assign modes by exploiting the information of users' velocity [66]. It indicates that users with higher velocity may allocate more frequency channels by using mode selection function. It is also suggested that the users of high mobility cover a large area as they move through space, while those users, having low mobility stay in a similar location. Therefore, more frequent channels can be allocated for higher velocity users to transmit more data than low mobility users in a random direction with uniform linear motion. In fact, user mobility is an inherent characteristic of the network to change the network topology over time scale in the mode selection.

\section{E. Role of Encounter Frequencies}

From the discussion in Section III, we have found that seminal papers showed that in a highly dynamic environment where frequent changes occurs in network topology can significantly increase the capacity or throughput of the user. It is also showed that mobility can provide an increase in the system capacity when users are mobile rather than fixed. Other works showed that faster diffusive behavior of mobile users lead to greater number of encounters. These encounters help to higher delivery ratio under same transmission range. The authors in different works studied the performance by adding different time intervals such as contact time and inter contact time to investigate the contact or encounter frequencies. It is concluded that pause time or inter contact time may lead to slower diffusion which in turn leads to lower throughput. However, throughput and delay are significantly affected by mobility characteristics of the nodes, especially when there is a pause time between the encounters of D2D users.

\section{Discussion}

We sketch the mobility models, traces and technical issues in a dynamic environment for D2D communication. We foreground the key lessons learned derived from the review of existing work, and distinguish open problems that merit in future investigation.

\section{A. Overview of Mobility Assisted D2D Communication}

We categorize the mobility models and traces adopted in D2D communication, summarized in Fig. 3, Fig. 4, Fig. 5, and 
Table I. The classification of mobility models is done according to the existing research work in D2D communication. We focus on mobility models and traces with regards to human and vehicle behavior according to their movement patterns [73], [210], [211], speed, geographic location [104], [212], social characteristics [53], [85], [213], stochastic data [214] and frequent visiting places [82]. Mobility models include random mobility model [61], [72], human mobility model [74], vehicular mobility model, dynamic graph model [97], social group based mobility model [5], [215] and geographic based mobility model. In addition the mobility traces are taken according to the opportunistic contacts, social connections, user interest, mobility and connectivity traces, vehicular network and local environment. We measuredly select the mobility models and traces from year 2009 up to 2018, in order to reflect form the old towards latest information regarding mobility models and traces.

After clear investigation in mobility models and traces in D2D communication, we outline the mobility aware fermented. According to the literature survey, we highlight the challenges with respect to target scenario, network interface, mobility models, mobility trace, number of devices, type of devices, and finally the issues due to the movements of human and vehicular. The summarization of different technical aspects of mobility assisted D2D communication is given in Table III-VII, respectively. We summarize diverse problems and their corresponding solutions due to mobility constraints. The summaries included in this paper shall furnish with a snapshot of the existing work consecrated to mobility assisted D2D communication. Therefore, our objective is to provide a comprehensive selection of existing work from 2012 till 2018 that can be leisurely for the interesting readers.

\section{B. Key Lessons Learned}

On the basis of reviewed papers, we derive a set of key lessons learned to be considering in the implementation of mobility in D2D communication.

1) Mobility Influence: There are several parameters that characterize the influence of mobility on D2D communication. For instance, a user enters the D2D coverage area in first time and leaves it in the second time. The probability that the user at the first time is within the D2D range of its proximate users, and is considered as contact probability. It determines the availability of D2D connectivity, and the duration of D2D link availability, and is called as CT. However, the time at which the mobile user leaves the range of D2D connectivity for certain duration is named as ICT.

Network topologies and users' are frequently changed in dynamic environments that ultimately affect the users' performance, e.g., data rates and latency. ICT is normally observed in human mobility as well as in vehicular mobility. This is because a person remains at a given location for a certain time period before moving to a new location. Similarly, vehicles can either stop at different locations due to road signals or move in different velocity with respect to other vehicles. Therefore, an ICT is the basic delay factor between the two targeted users being in contact with each other. However, sometimes the ICT can be large enough that the connectivity of D2D link can no longer be existed.

From the existing work, we learned that there is still a missing portion to effectively integrate mobility in D2D communication. For instance, the data downloading time and data dissemination depend on the size of data and the contact duration of the D2D link. The mobility of D2D users will not be in a positive mood if the data size is larger than the contact time of D2D link. In this case, the actual type of users' movement can insignificantly affect the ensuing system performance. It is believed that real time applications are strictly restraint with strict latency requirements, and therefore $\mathrm{CT}$ is the dominating mobility factor.

2) Users' Mobility Patterns: User mobility mostly depends on the spatial and temporal properties of D2D users. The spatial properties include the information of D2D user mobility pattern related to physical position. In addition, the temporal properties are related to time frame features. In fact, the mobility pattern of a mobile user can be envisioned by the D2D users' trajectories i.e., the user's moving path. The users' trajectories help in the D2D link establishment, and measures the distance between proximate mobile users. It is also noticed that user mobility patterns mostly depend on the social relationships among mobile users. For instance, the mobile users having relatively substantial social ties are more potential to have similar trajectories [76], [88], [104]. The user mobility patterns possesses a periodic property to tackle the adaptive caching and offloading [216], link establishment and content transmission problems.

Mobility patterns are necessary factors in a dynamic D2D communication environment [92], [163], [168]. However, some personal information may be unwrapped in the collection of mobility information to take the convinced advantages of mobility patterns. This personal information of mobile users includes home location, work place and daily life routines. This induces some dis-confirming concerns on the privacy issues of users. Thus, the extraction of user mobility information is significant without affecting user privacy. For this purpose, location mystification and fake location interposes techniques may serve as potential impending for anonymous mobility patterns [9], [53], [205].

3) Mobility in Caching and Offloading Design: D2D caching and offloading is not fixed, since they are related to the storage and dissemination of data usage of mobile users [169]. The data storage is different from user to user and may change over time. It is crucial to inquire the caching process according to the dynamic environment of user capabilities and mobility constraints. D2D links are found one of the best solutions for caching and offloading contents. Thus, the information associated with user contacts is indispensable for caching and offloading in the design settings. In this regard, some researchers leveraged the social phenomena for caching and offloading to disintegrate a large network into several small social groups. Therefore, it reduces the time complexity of caching and offloading design [206], [217]. In addition, the caching and offloading contents are adapted on the periodic patterns, for which the cognition of return times is utilitarian.

Nevertheless, users in a diverse social groups have different 
content preferences. Therefore, mobility pattern of each social group can be utilized to improve the adaptive caching design. For instance, in the proactive caching context, the future serving mobile node can be predicted, if the user pattern can be estimated based on the past status. In this scenario, if the user requests a certain file when passing by, the serving user (predicted to be on its future path) may proactively cache the file with a certain segment, and then the requester can download the file. This kind of situation may improve the caching efficiency and reduce the latency in downloading.

4) Time Altering Social-Interaction and Heterogeneous Human Walk: Social-interaction constantly covers large number of wirelessly connected movable people in a future society. However, it requires careful design and validation of prospective mobile networking technologies. To be specific, the scope of D2D communication is imperative in social context, which is to be covered beyond the best effort associations and services, support critical information exchange (i.e., road safety applications), dependability (i.e., timeliness) and reliability of data transmission with respect to mobility.

HHM generates the realistic users' pattern. It is based on the observation of real social networks or the realistic assumptions of an overlapping community structures. As a matter of fact, the popularity of users' pattern is categorized into real trace and social based aware i.e., online or offline social networks. However, the time varying social characters of people during a certain period of time impart to various social networks and corresponding overlapping community structures at versatile period [51], [85], [218], [218]. In result, an intuitional step is the first remark or artificially depicts a social graph by using a social network to obtain a realistic community structure.

Although social aware models are intensively attempted to find the social dimension causes by users' pattern, yet none of them consider how social network structure contributes to heterogeneous human popularity. Additionally, social aware models are based on manually stimulus or unnaturally generated social graphs that induce some serious troubles. First of all, it is inapplicable to manually input a social graph for mobility modeling, because the mobility model can only imitate the particular assumptions, and necessitates a great amount of attempt, when the imitation population is large. Secondly, the social aware models are not realistic enough in the aforesaid social aware mobility models [85], [219]-[221].

5) High Speed Vehicles: The goal of 3GPPs V2X-LTE (vehicular to $\mathrm{X}$ - long term evaluation) is to provide LTE support of vehicular communications. Such V2X specs allow generating revenue via high bandwidth infomercial applications for car users and proximity services [202]. It affirms the traffic telematics and ITS; equilibrating or even replacing IEEE $802.11 \mathrm{p}$ based consecrated short range communications. There are three technologies for the realization of LTE-based vehicular communications. (1) Dual connectivity to support high user mobility, (2) LTE-based disseminate services for effective distribution of messages among vehicles, and (3) Proximity based D2D communication is to actualize connectivity between vehicles as well as between connected cards and hand-held terminals [222].

Vehicular communication and RSU infrastructure can ex- change information about vehicles status, their location, and the road environment in cooperative ITS. In this regard, European Telecommunications Standard Institute (ETSI) defined cooperative awareness messages (CAM) and decentralized environmental notification messages (DENM) [223]. CAMs are periodically generated contents, that can be applied to exchange vehicle condition information with cars in the locality. It can be exchanged either directly or indirectly via dedicate short range communication utilizing the cellular network. The same case is used in U.S. Society of Automotive Engineers (SAE) J2735 standard, and is denoted by Basic Safety Message (BSM) [224]. On the other side, DENMs are event driven, and hence generated only periodically. For instance, in the case of emergency to warn upcoming vehicles, their distribution in certain notified areas must be efficiently handled. However, the geographical information is necessary to be considered.

Vehicles can exchange content with the aid of D2D communication. For example, a group of vehicles in the same area or in the same road can share data such as, traffic safety, road information, and traffic information by directly communicating with each other without putting the load on cellular network or RSU. Meanwhile, the moving vehicle can obtain the content from the parked vehicle in the specific spot by passing through that area, and can exchange content having similar interest. Thus, by enabling direct communication between vehicles, a large number of vehicles can exchange content with each other without relying on the RSU or any other centralized way.

6) Stochastic Geometry Implementation: Stochastic geometry is also considered in many research article to focus on the mobility behavior of users [214]. The modeling of stochastic geometry is the significant part of the D2D network design and analysis in the mobile premise. Different approaches show that stochastic geometry provides good tractability of the mobility behavior of D2D users and dynamic network topology. The basic idea in stochastic geometry is to find the Poisson point process (PPP) for the availability of users in the network deployment and coverage areas.

Stochastic geometry is considered to provide a good solution of randomly designed D2D communication to predict probabilistic parameters such as SINR distribution, coverage probability and load distribution [54], [198], [199]. Therefore, most of the work considerably achieved the performance of D2D communication in terms of throughput, QoS, SINR and data transmission by utilizing stochastic geometry [58], [115], [174], [204]. The research work showed that statistical analysis performed an appropriate mobility model and ensemble mobility parameters from mobility traces. The performance of D2D communication based on mobility traces collect the statistical data of CT and ICT. These statistical data include probability density function, cumulative distribution function, expected value or mean, and variance.

7) Mobility Benefits: It is a solid case to cautiously evaluating the existing challenges, assessing out the best solutions, detailing the resulting advantages, and finding the best technological solutions. The advantages of mobility stretch right across the operators, users, and all levels of resources. The following benefits can be achieved according to the lesson learned from the current research work. 
1) Efficient mobility management and remote resources.

2) Operational costs reduction.

3) Delivery services improvement.

4) Improved productivity.

5) Link continuity ensurance.

6) Gaining users' loyalty.

7) Attracting new users.

8) Flexible end users.

9) Sustain a competitive advantage.

The mobility solutions can provide all the above advantages if we implement the right decision for enabling communication and information access at anytime and anywhere.

\section{Open Problems}

The integration of mobility in D2D communication is broadly studied to solve different technical problems in the past few years. However, there are some special concerns for D2D communication owing to new requirements and use cases. Consequently, we list the open issues that deserve further investigation.

1) Mobility Management: Mobility management solutions are one of the basic requirements to minimize the negative impacts on D2D communication. Such negative impacts comprise of larger latency and additional signaling congestion in D2D communication. This can be achieved by controlling the D2D control handover and cell selection during mobile scenario. However, when two targeted users are active and they (either one or both) change their position, then it is not difficult to keep the service uninterrupted in the case of cellular mode. On the other side, when it is D2D mode, then mobility management become one of the essential problem that need to be taking care off. Mobility management become crucial in D2D mode and it requires proper algorithms to develop that can deal without interruption. Very few papers hashed out the mobility management in D2D communication and proper investigations and usage of proper algorithms are yet to develop to handle such problems.

2) User Experience: Data traffic performance substantially debauched as D2D users move at high speed. Subsequently, the data traffic is expected the fast signal overhead that cause congestion and ultimately admonishing user's experience. The mobility due to eminent devices can weaken the overall function. The growth of data traffic per device, and high end devices can increase the users' CT. For instance, the increase in mobile video content has much higher bit rates than other kind of mobile data. In result, larger CT is demanded between D2D users for video traffic, and need careful investigation. It is also worth noting that speed of users in terms of human walk or vehicles is the most interesting research direction because the speed can degrade or aggrade the link establishment between the users in a dynamic environment.

3) Mobility Prediction: Mobility prediction is an interesting research area, and is based on the recent history of users' trajectories. Mobility predictions and their comparability with empirical data may assist to realize human movement in a better way. It is influenced that we can be able to understand a lot about human mobility and can be represented mathematically, if human mobility is predicted within a given error percentage. People are often invoked to popular locations and the popularity of a given places importantly ascertains their frequency of visit to such areas. It is believed that mobility patterns can be easily collected in such kind of places, and therefore, sculptured an appropriate mobility model. Most of researchers have been used the available mobility traces for evaluating the performance of mobility aware D2D communication, but they fail to take the very true nature of mobility before intending new techniques.

4) Distributed Algorithms in Mobility: The high mobility of vehicular setup must be investigated in terms of cost and benefit of distributed algorithms for scheduling, resource allocation and interference control. Most of the existent techniques depend on a centralized execution. However, distributed algorithms can reduce the time cost and is a hot topic in future research directions. In the utmost case, the utterly distributed access could be the pure cognitive radio approach based on spectrum sensing [108]. In result, it gives in the interference controllability boasted by D2D communications. Consequently, it must be interesting topic to find out how one can attain for the best trade-off between centralized and distributed approaches while asserting an acceptable execution of variable ITS applications in intriguing vehicular scenarios. The study of D2D for ITS is a predicting technology that capable of encouraging the spectrum usage in vehicular applications. Therefore, it is a challenging problem that awaits innovative yet practical solutions.

5) Geographical and Network Information: It is also an interesting research direction to obtain data that contain the geographical and network information at the same time. None of the existing work indicates the strong correlation between geographical and network information that emerges in many diverse techniques. This kind of information represented the similarity between users' movements, and the effectiveness of interaction. Human mobility in their geographical location serve as a good prognosticator for the information of new D2D links, that yields comparable predictive power as compared to traditional network based measurements. In addition, by aggregating both mobility and network measurements, we can be able to find the prediction accuracy that can be significantly improved in the supervised acquisition. Finally, another interesting direction is to uncover the strong correlation-ship between mobility similarity, social connection, and prediction.

6) Requirement Gaps and Conflicts: Several mobility models are failed to achieve the true nature of mobility analysis, and therefore, it is not applicable to incorporate in D2D communication. These mobility models do not reflect the true nature of mobility as ascertained in real life. Random way point has its inherent anomalousness, while random direction is delusive, because it is improbable that users would distribute themselves evenly across an area. Similarly, some models are difficult to investigate by selecting the right parameters. Therefore, one needs to select an appropriate mobility model to capture the realistic mobility patterns, and human or vehicle real movements. Hence, it is one the most important research direction in the implementation of mobility.

Moreover, the traces used by most of researchers are still deprived of real life scenario. It is strongly recommend that 
researchers should unfold the recent data traces to investigate the mobility effect on users and system performance. It is also encouraged to find out the contact duration, inter-contact time, number of encounters, and their encounters' location from the chosen realistic traces, simultaneously. Since most of the authors did not mention different mobility parameters simultaneously in their work such as, type of network interface, mobility traces, and number of devices, contact frequency, type of devices, and corresponding speed.

7) Macroscopic Mobility: Heterogeneous Random Walk (HRW) captures the properties of macroscopic mobility model [225]. Heterogeneous spatial node distribution is one such macroscopic property. Heterogeneity of HRW model originates due to difference in users' speed and dynamics of connectivity. It also provides information about traffic trends of all vehicles in a city and helps in path selection.

\section{CONCLUSION}

We conceive that our work furnishes detailed survey and state-of-the-art researches in mobility and its impact on D2D communication. We have presented a broad research on the subject and have foregrounded the major findings to date. We carve up the survey into three major sections. The first part provides a detailed version of mobility models and traces that are adopted or can be used in D2D communication. The second part of the paper deals with technical problems and its solutions. Furthermore, the impact of mobility in D2D communication is also depicted that will help the readers and researchers to investigate how mobility will help in D2D communication. In addition, we derive the key lesson learned and distinguish open problems that merit future research directions. We believe that the discussion presented in this review will suffice as a reference guide for researchers and developers to facilitate the design and implementation of mobility aware D2D communication.

\section{REFERENCES}

[1] M. Agiwal, A. Roy, and N. Saxena, "Next generation 5G wireless networks: A comprehensive survey," IEEE Commun. Surveys \& Tut., vol. 18, no. 3, pp. 1617-1655, Thirdquarter 2016.

[2] H. Li, B. Wang, Y. Song, and K. Ramamritham, "VeShare: A D2D infrastructure for real-time social-enabled vehicle networks," IEEE Wireless Commun., vol. 23, no. 4, pp. 96-102, Aug. 2016.

[3] A. Nshimiyimana, D. Agrawal, and W. Arif, "Comprehensive survey of V2V communication for 4G mobile and wireless technology," in Proc. WiSPNET 2016 (Chennai, India), Mar. 23-25, 2016, pp. 1722-1726.

[4] H. Pang, et al., "Crowdsourced mobility prediction based on spatiotemporal contexts," in Proc. ICC 2016 (Kuala Lumpur, Malaysia), May 23-27, 2016, pp. 1-6.

[5] S. Schwarz and M. Rupp, "Society in motion: Challenges for LTE and beyond mobile communications," IEEE Commun. Mag., vol. 54, no.5, pp. 76-83, May 2016.

[6] B. Romanous, N. Bitar, A. Imran, and H. Refai, "Network densification: Challenges and opportunities in enabling 5G," in Proc. CAMAD 2015 (Guildford, UK), Sep. 7-9, 2015, pp. 129-134.

[7] Y. Yang, et al., "Link prediction in human mobility networks," in Proc. ASONAM 2013 (Niagara Falls, Canada), Aug. 25-28, 2013, pp. 380-387.

[8] A. Asadi, Q. Wang, and V. Mancuso, "A survey on device-to-device communication in cellular networks," IEEE Commun. Surveys Tut., vol. 16, no. 4, pp. 1801-1819, Fourthquarter 2014.

[9] M. Haus, et al., "Security and privacy in device-to-device (D2D) communication: A review," IEEE Commun. Surveys Tut., vol. 19, no. 2 , pp 1054-1079, Secondquarter 2017.
[10] P. Mach, Z. Becvar, and T. Vanek, "In-band device-to-device communication in OFDMA cellular networks: A survey and challenges," IEEE Commun. Surveys Tut., vol. 17, no. 4, pp. 1885-1922, Fourthquarter 2015.

[11] J. Liu, N. Kato, J. Ma, and N. Kadowaki, "Device-to-device communication in LTE-advanced networks: A survey," IEEE Commun. Surveys Tut., vol. 17, no. 4, pp. 1923-1940, Fourthquarter 2015.

[12] H. A. U. Mustafa, et al., "Separation framework: An enabler for cooperative and D2D communication for future 5G networks," IEEE Commun. Surveys Tut., vol. 18, no. 1, pp. 419-445, Firstquarter 2016.

[13] R. Alkurd, R. M. Shubair, and I. Abualhaol, "Survey on device-to-device communications: Challenges and design issues," in Proc. NEWCAS 2014 (Trois-Rivieres, Canada), Jun. 22-25, 2014, pp. 361-364.

[14] M. Wang and Z. Yan, "Security in D2D communications: A review," in Proc. 2015 IEEE Trustcom/BigDataSE/ISPA (Helsinki, Finland), Aug. 20-22, 2015, pp. 1199-1204.

[15] M. Noura and R. Nordin, "A survey on interference management for D2D communication and its challenges in 5G networks," J. Network and Computer Applications, vol. 71, pp. 130-150, Aug. 2016.

[16] M. Ahmed, et al., "A survey on socially aware device-to-device communications," IEEE Commun. Surveys Tut., vol. 20, no. 3, pp. 2169-2197, Thirdquarter 2018.

[17] Z. M. Fadlullah, et al., "Toward intelligent machine-to-machine communications in smart grid," IEEE Comm. Mag., vol. 49, no. 4, pp. 60-65, Apr. 2011

[18] S. Chen, et al., "Machine-to-machine communications in ultra-dense networks - a survey," IEEE Commun. Surveys Tut., vol. 19, no. 3 , pp. 1478-1503, Thirdquarter 2017.

[19] M. A. Mehaseb, Y. Gadallah, A. Elhamy, and H. Elhennawy, "Classification of LTE uplink scheduling techniques: An M2M perspective," IEEE Commun. Surveys Tut., vol. 18, no. 2, pp. 1310-1335, Secondquarter 2016.

[20] M. Chen, et al., "A survey of recent developments in home M2M networks," IEEE Commun. Surveys Tut., vol. 16, no. 1, pp. 98-114, Firstquarter 2014

[21] A. Barki, A. Bouabdallah, S. Gharout, and J. Traoré, "M2M security: Challenges and solutions," IEEE Commun. Surveys Tut., vol. 18, no. 2, pp. 1241-1254, Secondquarter 2016.

[22] J. Kim, J. Lee, J. Kim, and J. Yun, "M2M service platforms: Survey, issues, and enabling technologies," IEEE Commun. Surveys Tut., vol. 16, no. 1, pp. 61-76, Firstquarter 2014.

[23] M. Jo, et al., "A survey of converging solutions for heterogeneous mobile networks," IEEE Wireless Commun., vol. 21, no. 6, pp. 54-62, Dec. 2014.

[24] A. Rajandekar and B. Sikdar, "A survey of MAC layer issues and protocols for machine-to-machine communications," IEEE Internet of Things J., vol. 2, no. 2, pp. 175-186, Apr. 2015.

[25] Y. Cao, T. Jiang, and Z. Han, "A survey of emerging M2M systems: Context, task, and objective," IEEE Internet of Things J., vol. 3, no. 6 , pp. 1246-1258, Dec. 2016.

[26] E. Soltanmohammadi, K. Ghavami, and M. Naraghi-Pour, "A survey of traffic issues in machine-to-machine communications over LTE," IEEE Internet of Things $J$. , vol. 3, no. 6, pp. 865-884, Dec. 2016.

[27] M. Waqas, et al., "Social-aware secret key generation for secure deviceto-device communication via trusted and non-trusted relays," IEEE Trans. Wireless Commun., vol. 17, no. 6, pp. 3918-3930, Jun. 2018.

[28] M. Ahmed, et al., "Socially aware secrecy-ensured resource allocation in D2D underlay communication: An overlapping coalitional game scheme," IEEE Trans. Wireless Commun., vol. 17, no. 6, pp. 4118-4133, Jun., 2018.

[29] Y. Li, D. Jin, P. Hui, and S. Chen, "Contact-aware data replication in roadside unit aided vehicular opportunistic networks," IEEE Trans. Mobile Computing, vol. 15, no. 2, pp. 306-321, Feb. 2016

[30] S. Chen, et al., "Vehicle-to-everything (V2X) services supported by LTE-based systems and 5G," IEEE Commun. Standards Mag., vol. 1, no. 2, pp. 70-76, 2017.

[31] K. Zheng, et al., "Heterogeneous vehicular networking: A survey on architecture, challenges, and solutions," IEEE Commun. Surveys Tut., vol. 17, no. 4, pp. 2377-2396, Fourthquarter 2015.

[32] B. B. Rhoades and J. M. Conrad, "A survey of alternate methods and implementations of an intelligent transportation system," in Proc. SoutheastCon 2017 (Charlotte, USA), Mar. 30-Apr. 2, 2017, pp. 1-8.

[33] J. Rios-Torres and A. A. Malikopoulos, "A survey on the coordination of connected and automated vehicles at intersections and merging at highway on-ramps," IEEE Trans. Intelligent Transportation Systems, vol. 18 , no. 5, pp. 1066-1077, May 2017. 
[34] S. Glass, I. Mahgoub, and M. Rathod, "Leveraging MANET-based cooperative cache discovery techniques in VANETs: A survey and analysis," IEEE Commun. Surveys Tut., vol. 19, no. 4, pp. 2640-2661, Fourthquarter 2017.

[35] A. Nadeem and M. P. Howarth, "A survey of MANET intrusion detection \& prevention approaches for network layer attacks," IEEE Commun. Surveys Tut., vol. 15, no. 4, pp. 2027-2045, Fourthquarter 2013.

[36] T. R. Andel and A. Yasinsac, "Surveying security analysis techniques in MANET routing protocols," IEEE Commun. Surveys Tut., vol. 9, no. 4, pp. 70-84, Fourthquarter 2007.

[37] J. Luo, D. Ye, X. Liu, and M. Fan, "A survey of multicast routing protocols for mobile ad-hoc networks," IEEE Commun. Surveys Tut., vol. 11, no. 1, pp. 78-91, Firstquarter 2009.

[38] Y. Li, P. Hui, D. Jin, and S. Chen, "Delay-tolerant network protocol testing and evaluation," IEEE Commun. Mag., vol. 53, no. 1, pp. 258 266, Jan. 2015.

[39] P. U. Tournoux, et al., "Density-aware routing in highly dynamic DTNs: The RollerNet case," IEEE Trans. Mobile Computing, vol. 10, no. 12, pp. 1755-1768, Dec. 2011

[40] M. H. Zayani, V. Gauthier, I. Slama, and D. Zeghlache, "Tracking topology dynamicity for link prediction in intermittently connected wireless networks," in Proc. IWCMC 2012 (Limassol, Cyprus), Aug. 27-31, 2012, pp. 469-474.

[41] Y. Li, et al., "The impact of node selfishness on multicasting in delay tolerant networks," IEEE Trans. Veh. Techno., vol. 60, no. 5, pp. 2224 2238, Jun. 2011.

[42] Y. Li, et al., "An optimal relaying scheme for delay-tolerant networks with heterogeneous mobile nodes," IEEE Trans. Veh. Techno., vol. 62 no. 5, pp. 2239-2252, Jun. 2013.

[43] W. Ren, et al., "Optimal vehicles and coding decision for mobile data sharing in vehicular delay tolerant networks," in Proc. ICNP 2012 (Austin, USA), Oct. 31-Nov. 2, 2012, pp. 1-2.

[44] H. Zhang, et al., "Buffer-aided D2D communications: Opportunities and challenges," IEEE Commun. Mag., vol. 53, no. 12, pp. 67-74, Dec. 2015.

[45] F. Wang, Z. Wang, Z. Yang, and S. Chen, "Contact duration aware cache refreshing for mobile opportunistic networks," IET Networks, vol. 5, no. 4, pp. 93-103, 2016.

[46] C. Gao, Y. Li, Y. Zhao, and S. Chen, "A two-level game theory approach for joint relay selection and resource allocation in network coding assisted D2D communications," IEEE Trans. Mobile Computing, vol. 16, no. 10 , pp. 2697-2711, Oct. 2017

[47] D. Xu, et al. "A survey of opportunistic offloading," IEEE Commun. Surveys Tut., vol. 20, no. 3, pp. 2190-2236, Thirdquarter 2018.

[48] X. Chen, et al. "Social trust aided D2D communications: Performance bound and implementation mechanism," IEEE J. Sel. Areas Commun., vol. 36, no. 7, pp. 1593-1608, Jul. 2018

[49] S. Batabyal and P. Bhaumik, "Mobility models, traces and impact of mobility on opportunistic routing algorithms: A survey," IEEE Commun. Surveys Tut., vol. 17, no. 3, pp. 1679-1707, Thirdquarter 2015.

[50] T. Camp, J. Boleng, and V. Davies, "A survey of mobility models for ad hoc network research," Wireless Commun. Mobile Computing, vol. 2 , no. 5, pp. 483-502, 2002.

[51] A. Orsino, et al., "Effects of heterogeneous mobility on D2D- and droneassisted mission-critical MTC in 5G," IEEE Commun. Mag., vol. 55, no. 2, pp. 79-87, Feb. 2017.

[52] S. Laqtib, et al., "Impact of mobility models on optimized link state routing protocol in MANET," in Proc. WINCOM 2016 (Fez, Morocco), Oct. 26-29, 2016, pp. 104-109.

[53] H. Lenando, M. N. Jambli, K. Zen, and J. Abdullah, "Impact of mobility models on social structure formation in opportunistic networks," in Proc. 12th IEEE Int. Conf. Trust, Security and Privacy in Computing and Commun. (Melbourne, Australia), Jul. 16-18, 2013, pp. 1434-1441.

[54] A. Orsino, et al., "Assisted handover based on device-to-device communications in 3GPP LTE systems," in Proc. Globecom 2015 Workshops (San Diego, USA), Dec. 6-10, 2015, pp. 1-6.

[55] R. Lan, W. Wang, A. Huang, and H. Shan, "Device-to-device offloading with proactive caching in mobile cellular networks," in Proc. Globecom 2015 (San Diego, USA), Dec. 6-10, 2015, pp. 1-6.

[56] D. Wu, Y. Cai, R. Q. Hu, and Y. Qian, "Dynamic distributed resource sharing for mobile D2D communications," IEEE Trans. Wireless Commun., vol. 14, no. 10, pp. 5417-5429, Oct. 2015.

[57] B. Zhang, et al., "Social-aware peer discovery for D2D communications underlaying cellular networks," IEEE Trans. Wireless Commun., vol. 14 no. 5, pp. 2426-2439, May 2015.

[58] X. Xu, et al., "Analytical modeling of mode selection for moving D2D-enabled cellular networks," IEEE Commun. Let., vol. 20, no. 6 , pp. 1203-1206, Jun. 2016.
[59] B. Panigrahi, R. Ramamohan, H. K. Rath, and A. Simha, "Dynamic relay selection and channel adaptive uplink for LTE device-to-device (D2D) communication," in Proc. PIMRC 2016 (Valencia, Spain), Sep. 4-8, 2016, pp. 1-6.

[60] J. Yoon, M. Liu, and B. Noble, "Random waypoint considered harmful," in Proc. INFOCOM 2003 (San Francisco, USA), Mar. 30-Apr. 3, 2003, pp. 1312-1321.

[61] R. R. Colletti and R. M. de Moraes, "Evaluation of link lifetime for the random waypoint mobility model," in Proc. ICON 2013 (Singapore), Dec. 11-13, 2013, pp. 1-5.

[62] C. Bettstetter, "Mobility modeling in wireless networks: categorization, smooth movement, and border effects," ACM SIGMOBILE Mobile Computing and Commun. Review, vol. 5, no. 3, pp. 55-66, Jul. 2001.

[63] D. M. Blough, G. Resta, and P. Santi, "A statistical analysis of the long-run node spatial distribution in mobile ad hoc networks," Wireless Networks, vol. 10, no. 5, pp. 543-554, Sep. 2004.

[64] E. M. Royer, P. M. Melliar-Smith, and L. E. Moser, "An analysis of the optimum node density for ad hoc mobile networks," in Proc. ICC 2001 (Helsinki, Finland), Jun. 11-14, 2001, pp. 857-861.

[65] H.-D. Bae, J.-W. Shin, and P.-J. Song, "Mode switching for deviceto-device communication in LTE-A network," in Proc. MoMM 2014 (Kaohsiung, Taiwan, China), Dec. 8-10, 2014, pp. 7-10.

[66] H. Kuribayashi, et al., "A mobility-based mode selection technique for fair spatial dissemination of data in multi-channel device-to-device communication," in Proc. ICC 2016 (Kuala Lumpur, Malaysia), May 22-27, 2016, pp. 1-6.

[67] K. W. Choi, D. T. Wiriaatmadja, and E. Hossain, "Discovering mobile applications in cellular device-to-device communications: Hash function and bloom filter-based approach," IEEE Trans. Mobile Computing, vol. 15, no. 2, pp. 336-349, Feb. 2016.

[68] H. Zhang, P. Hong, and K. Xue, "Mobile-based relay selection schemes for multi-hop cellular networks," J. Commun. and Networks, vol. 15 , no. 1, pp. 45-53, Feb. 2013.

[69] S. Hosny, A. Eryilmaz, and H. E. Gamal, "Impact of user mobility on D2D caching networks," in Proc. Globecom 2016 (Washington DC, USA), Dec. 4-8, 2016, pp. 1-6.

[70] S. Hosny, A. Eryilmaz, A. A. Abouzeid, and H. E. Gamal, "Mobilityaware centralized D2D caching networks," in Proc. 54th Annual Allerton Conf. Commun., Control, and Computing (Monticello, USA), Sep. 2730, 2016, pp. 725-732.

[71] Y. Liu, et al., "Multi-copy data dissemination with probabilistic delay constraint in mobile opportunistic device-to-device networks," in Proc. WoWMoM 2016 (Coimbra, Portugal), Jun. 21-24, 2016, pp. 1-9.

[72] J. Zhang, et al., "Analysis of random walk mobility models with location heterogeneity," IEEE Trans. Parallel and Distributed Systems, vol. 26, no. 10 , pp. 2657-2670, Oct. 2015.

[73] J. Zhang, L. Fu, X. Wang, and M. Guizani, "Impact of location heterogeneity on random walk mobility models," in Proc. Globecom 2013 (Atlanta, USA), Dec. 9-13, 2013, pp. 365-370.

[74] D. Karamshuk, C. Boldrini, M. Conti, and A. Passarella, "Human mobility models for opportunistic networks," IEEE Commun. Mag., vol. 49, no. 12, pp. 157-165, Dec. 2011

[75] D. Brockmann, L. Hufnagel, and T. Geisel, "The scaling laws of human travel," Nature, vol. 439, no. 7075, pp. 462-465, 2006.

[76] M. C. Gonzalez, C. A. Hidalgo, and A.-L. Barabasi, "Understanding individual human mobility patterns," Nature, vol. 453, no. 7196, pp. 779782,2008

[77] C. Song, T. Koren, P. Wang, and A.-L. Barabási, "Modelling the scaling properties of human mobility," Nature Physics, vol. 6, no. 10, pp. 818823,2010

[78] A. Chaintreau, et al., "Impact of human mobility on opportunistic forwarding algorithms," IEEE Trans. Mobile Computing, vol. 6, no. 6, pp. 606-620, Jun. 2007.

[79] T. Karagiannis, J. Y. Le Boudec, and M. Vojnovic, "Power law and exponential decay of intercontact times between mobile devices," IEEE Trans. Mobile Computing, vol. 9, no. 10, pp. 1377-1390, Oct. 2010.

[80] P. Hui and J. Crowcroft, "Human mobility models and opportunistic communications system design," Philos. Trans. A Math. Phys. Eng. Sci., vol. 366 , no. 1872 , pp. 2005-2016, 2008.

[81] K. Lee, et al., "SLAW: A new mobility model for human walks," in Proc. INFOCOM 2009 (Rio de Janeiro, Brazil), Apr. 19-25, 2009, pp. 855863.

[82] G. Solmaz, M. A. Akbar, and D. Turgut, "A mobility model of theme park visitors," IEEE Trans. Mobile Computing, vol. 14, no. 12, pp. 24062418, Dec. 2015. 
[83] M. A. Alim, T. Pan, M. T. Thai, and W. Saad, "Leveraging social communities for optimizing cellular D2D communications," IEEE Trans. Wireless Commun., vol. 16, no. 1, pp. 551-564, Jan. 2017.

[84] M. Gorawski and K. Grochla, "The real-life mobility model: RLMM," in Proc. FGCT 2013 (London, UK), Dec. 12-14, 2013, pp. 201-206.

[85] S. Yang, X. Yang, C. Zhang, and E. Spyrou, "Using social network theory for modeling human mobility," IEEE Network, vol. 24, no. 5, pp. 6-13, Sep. 2010.

[86] L. Karim and Q. H. Mahmoud, "A hybrid mobility model based on social, cultural and language diversity," in Proc. 9th IEEE Int. Conf. Collaborative Computing: Networking, Applications and Worksharing (Austin, USA), Oct. 20-23, 2013, pp. 197-204.

[87] C. Wang, Y. Li, and D. Jin, "Mobility-assisted opportunistic computation offloading," IEEE Commun. Let., vol. 18, no. 10, pp. 1779-1782, Oct. 2014.

[88] E. Baștuğ, M. Bennis, and M. Debbah, "Social and spatial proactive caching for mobile data offloading," in Proc. ICC 2014 Workshops (Sydney, Australia), Jun. 10-14, 2014, pp. 581-586.

[89] M. Musolesi and C. Mascolo, "Designing mobility models based on social network theory," ACM SIGMOBILE Mobile Computing and Commun. Review, vol. 11, no. 3, pp. 59-70, Jul. 2007.

[90] Y. Li, et al., "Social-aware D2D communications: Qualitative insights and quantitative analysis," IEEE Commun. Mag., vol. 52, no. 6, pp. 150158, Jun. 2014

[91] M. Musolesi, S. Hailes, and C. Mascolo, "An ad hoc mobility model founded on social network theory," in Proc. MSWiM 2004 (Venice, Italy), Oct. 4-6, 2004, pp. 20-24.

[92] C. M. Huang, C. H. Lee, and H. Y. Lai, "Energy-aware group LBS using D2D offloading and M2M-based mobile proxy handoff mechanisms over the mobile converged networks," IEEE Transa. Emerging Topics Computing, vol. 4, no. 4, pp. 528-540, Oct.-Dec. 2016.

[93] T. Wang, Y. Sun, L. Song, and Z. Han, "Social data offloading in D2Denhanced cellular networks by network formation games," IEEE Trans. Wireless Commun., vol. 14, no. 12, pp. 7004-7015, Dec. 2015.

[94] F. Harary and G. Gupta, "Dynamic graph models," Mathematical and Computer Modelling, vol. 25, no. 7, pp. 79-87, Apr. 1997.

[95] F. Malandrino, C. Casetti, C. F. Chiasserini, and M. Fiore, "Content downloading in vehicular networks: What really matters," in Proc. INFOCOM 2011 (Shanghai, China), Apr. 10-15, 2011, pp. 426-430.

[96] Y. Li, D. Jin, P. Hui, and Z. Han, "Optimal base station scheduling for device-to-device communication underlaying cellular networks," IEEE J. Selected Areas Commun., vol. 34, no. 1, pp. 27-40, Jan. 2016.

[97] Y. Li, C. Song, D. Jin, and S. Chen, "A dynamic graph optimization framework for multihop device-to-device communication underlaying cellular networks," IEEE Wireless Commun., vol. 21, no. 5, pp. 52-61, Oct. 2014

[98] Y. Li, Z. Wang, D. Jin, and S. Chen, "Optimal mobile content downloading in device-to-device communication underlaying cellular networks," IEEE Trans. Wireless Commun., vol. 13, no. 7, pp. 3596-3608, Jul. 2014

[99] Y. Zhao, et al., "Fundamental tradeoffs on energy-aware D2D communication underlaying cellular networks: A dynamic graph approach," IEEE J. Selected Areas Commun., vol. 34, no. 4, pp. 864-882, Apr. 2016.

[100] J. Tian, et al., "Graph-based mobility model for mobile ad hoc network simulation," in Proc. 35th Annual Simulation Symp. (San Diego, USA), Apr. 14-18, 2002, pp. 337-344.

[101] A. Morattab, Z. Dziong, K. Sohraby, and M. H. Islam, "Mobility impact on mode selection map in D2D networks; An analytical approach," in Proc. WCNC 2016 (Doha, Qatar), Apr. 3-6, 2016, pp. 1-6.

[102] F. Tian, B. Liu, J. Xiong, and L. Gui, "Movement-based incentive for cellular traffic offloading through D2D communications," in Proc. BMSB 2016 (Nara, Japan), Jun. 1-3, 2016, pp. 1-5.

[103] M. Zeng, et al., "Incentive mechanism design for computation offloading in heterogeneous fog computing: A contract-based approach," in Proc. ICC (Kansas, USA), pp. 1-6, May 20-24, 2018 ,

[104] D. Wang, et al., "Human mobility, social ties, and link prediction," in Proc. KDD 2011 (San Diego, USA), Apr. 21-24, 2011, pp. 1100-1108.

[105] UN, "World Urbanization Prospects [highlights], 2014 revision," 2014, esa. un.org/unpd/wup/.

[106] N. Eagle, A. S. Pentland, and D. Lazer, "Inferring friendship network structure by using mobile phone data," Proc. National Acad. Sci. USA, vol. 106, no. 36, pp. 15274-15278, 2009.

[107] S. C. Nelson, A. F. Harris III, and R. Kravets, "Event-driven, rolebased mobility in disaster recovery networks," in Proc. CHANTS 2007 (Montreal, Canada), Sep. 14, 2007, pp. 27-34.

[108] X. Cheng, L. Yang, and X. Shen, "D2D for intelligent transportation systems: A feasibility study," IEEE Trans. Intelligent Transportation Systems, vol. 16, no. 4, pp. 1784-1793, Aug 2015.
[109] A. Khelil and D. Soldani, "On the suitability of device-to-device communications for road traffic safety," in Proc. WF-IoT 2014 (Seoul, South Korea), Mar. 6-8, 2014, pp. 224-229.

[110] W. Sun, et al., "Radio resource management for D2D-based V2V communication," IEEE Trans. Veh. Techno., vol. 65, no. 8, pp. 66366650, Aug. 2016.

[111] M. Botsov, M. Klügel, W. Kellerer, and P. Fertl, "Location dependent resource allocation for mobile device-to-device communications," in Proc. WCNC 2014 (Istanbul, Turkey), Apr. 6-9, 2014, pp. 1679-1684.

[112] W. Xing, et al., "Resource allocation schemes for D2D communication used in VANETs," in Proc. VTC 2014-Fall (Vancouver, Canada), Sep. 14-17, 2014, pp. 1-6.

[113] W. Sun, et al., "D2D-based V2V communications with latency and reliability constraints," in Proc. Globecom 2014 Workshops (Austin, USA), Dec. 8-12, 2014, pp. 1414-1419.

[114] P. Kolios, K. Papadaki, and V. Friderikos, "Efficient cellular load balancing through mobility-enriched vehicular communications," IEEE Trans. Intelligent Transportation Systems, vol. 17, no. 10, pp. 29712983, Oct. 2016.

[115] L. Lei, "Stochastic modeling of device-to-device communications for intelligent transportation systems," in Proc. ICT 2016 (Thessaloniki, Greece), May 16-18, 2016, pp. 1-5.

[116] J. Barrachina, et al., "Road side unit deployment: A density-based approach," IEEE Intelligent Transportation Systems Mag., vol. 5, no. 3, pp. 30-39, Fall 2013.

[117] M. Barth and K. Boriboonsomsin, "Energy and emissions impacts of a freeway-based dynamic eco-driving system," Transportation Research Part D: Transport and Environment, vol. 14, no. 6, pp. 400-410, Aug. 2009.

[118] A. Halati, H. Lieu, and S. Walker, "CORSIM - corridor traffic simulation model," in Proc. Traffic Congestion and Traffic Safety in the 21st Century: Challenges, Innovations, and Opportunities (Chicago, USA), Jun. 8-11, 1997, pp. 570-576.

[119] A. P. Arias, et al., "A framework for evaluating the VISSIM traffic simulation with extended range telepresence," in Proc. CASA 2009 (Amsterdam, Netherlands), Jun. 17-19, 2009, pp. 13-16.

[120] "PTV Vissim," Simulation Software, PTV Group. https://www.ptvgroup.com/en/solutions/products/ptv-vissim/

[121] J. Barceló (ed.), Fundamentals of Traffic Simulation. Springer: New York, 2010.

[122] L. Smith, et al., "TRANSIMS: Transportation analysis and simulation system," Los Alamos National Lab., NM, USA, Tech. Rep., 1995.

[123] R. W. Marx, "The TIGER system: Automating the geographic structure of the united states census," Government Publications Review, vol. 13, no. 2, pp. 181-201, 1986

[124] STRAW - STreet RAndom Waypoint - vehicular mobility model for network simulations, AquaLab.

http://www.aqualab.cs.northwestern.edu/projects/144-straw-streetrandom-waypoint-vehicular-mobility-model-for-network-simulations-eg-car-networks

[125] S. Zeadally, et al., "Vehicular ad hoc networks (VANETs): Status, results, and challenges," Telecommunication Systems, vol. 50, no. 4 , pp. 217-241, 2012.

[126] M. Behrisch, L. Bieker, J. Erdmann, and D. Krajzewicz, "SUMO simulation of urban mobility: An overview," in Proc. SIMUL 2011 (Barcelona, Spain), Oct. 23-29, 2011, pp. 1-6.

[127] D. Krajzewicz, G. Hertkorn, C. Rössel, and P. Wagner, "SUMO (simulation of urban mobility) - an open-source traffic simulation," in Proc. MESM 2002 (Sharjah, UAE), Oct. 28-30, 2002, pp. 183-187.

[128] N. Eagle and A. S. Pentland, "CRAWDAD dataset mit/reality (v. 200507-01)," Jul. 2005. https://crawdad.org/mit/reality/20050701/

[129] J. Scott, et al., "CRAWDAD dataset cambridge/haggle (v. 2009-0529)", May 2009. https://crawdad.org/cambridge/haggle/20090529/

[130] M. Han, et al.,, "CRAWDAD dataset kaist/wibro (v. 2008-06-04)," Jun. 2008. https://crawdad.org/kaist/wibro/20080604/

[131] K. Papagiannaki, M. Yarvis, and W. S. Conner, "CRAWDAD dataset intel/home (v. 2006-04-16)," Apr. 2006. https://crawdad.org/intel/home/20060416/

[132] A.-K. Pietilainen and C. Diot, "CRAWDAD dataset thlab/sigcomm2009 (v. 2012-07-15)," Jul. 2012. https://crawdad.org/thlab/sigcomm2009/20120715/

[133] L. Bracciale, et al., "CRAWDAD dataset roma/taxi (v. 2014-07-17)," Jul. 2014. https://crawdad.org/roma/taxi/20140717/

[134] J. Burgess, et al., "CRAWDAD dataset umass/diesel (v. 2008-09-14)," Sep. 2008. https://crawdad.org/umass/diesel/20080914/ 
[135] D. Kotz, T. Henderson, I. Abyzov, and J. Yeo, "CRAWDAD dataset dartmouth/campus (v. 2009-09-09)," Sep. 2009. https://crawdad.org/dartmouth/campus/20090909/

[136] H. Zhu, et al., "Recognizing exponential inter-contact time in VANETs," in Proc. INFOCOM 2010 (San Diego, USA), Mar. 14-19, 2010, pp. 101-105.

[137] Y. Li, D. Jin, L. Zeng, and S. Chen, "Revealing patterns of opportunistic contact durations and intervals for large scale urban vehicular mobility," in Proc. ICC 2013 (Budapest, Hungary), Jun. 9-13, 2013, pp. 16461650.

[138] R. I. Ciobanu and C. Dobre, "CRAWDAD dataset upb/hyccups (v. 2016-10-17),’Oct. 2016, traceset: 2012. https://crawdad.org/upb/hyccups/20161017/

[139] D.-G. Akestoridis, "CRAWDAD dataset uoi/haggle (v. 2016-08-28): derived from cambridge/haggle (v. 2009-05-29),” Aug. 2016. http://crawdad.org/uoi/haggle/20160828

[140] S. Cabrero, R. García, X. G. García, and D. Melendi, "CRAWDAD dataset oviedo/asturies-er (v. 2016-08-08)," Aug. 2016. http://crawdad.org/oviedo/asturies-er/20160808

[141] S. Firdose, et al., "CRAWDAD dataset copelabs/usense (v. 2017-0127)," Jan. 2017. http://crawdad.org/copelabs/usense/20170127

[142] R. Parasuraman, S. Caccamo, F. Baberg, and P. Ogren, "CRAWDAD dataset kth/rss (v. 2016-01-05)," Jan. 2016. http://crawdad.org/kth/rss/20160105

[143] A. Caputo, A. Socievole, and F. D. Rango, "CRAWDAD dataset unical/socialblueconn (v. 2015-02-08)," Feb. 2015 http://crawdad.org/unical/socialblueconn/20150208

[144] A. Galati and C. Greenhalgh, "CRAWDAD dataset nottingham/mall (v 2013-02-05)," Feb. 2013. http://crawdad.org/nottingham/mall/20130205

[145] J. M. Cabero, et al., "CRAWDAD dataset tecnalia/humanet (v. 2012 06-12),” Jun. 2012. http://crawdad.org/tecnalia/humanet/20120612

[146] D. Wu, et al., "Social attribute aware incentive mechanism for deviceto-device video distribution," IEEE Trans. Multimedia, vol. 19, no. 8 , pp.1908-1920, Aug. 2017.

[147] Z. Wang, H. Shah-Mansouri, and V. W. S. Wong, "How to download more data from neighbors? A metric for D2D data offloading opportunity," IEEE Trans. Mobile Computing, vol. 16, no. 6, pp. 1658-1675, Jun., 2017.

[148] B. Baron, et al., "Centrally-controlled mass data offloading using vehicular traffic," IEEE Trans. Network and Service Management, vol. 14 no. 2, pp. 401-415, Jun. 2017.

[149] K. Chen, H. Shen, and L. Yan, "DSearching: Using floating mobility information for distributed node searching in DTNs," IEEE Trans. Mobile Computing, vol. 15, no. 1, pp. 121-136, Jan. 2016.

[150] K. Chen and H. Shen, "DSearching: Distributed searching of mobile nodes in DTNs with floating mobility information," in Proc. INFOCOM 2014 (Toronto, Canada), Apr. 27-May 2, 2014, pp. 2283-2291.

[151] K. Zhu, W. Li, and X. Fu, "SMART: A social- and mobile-aware routing strategy for disruption-tolerant networks," IEEE Trans. Veh. Techno., vol. 63, no. 7, pp. 3423-3434, Sep. 2014

[152] I. Rhee, et al., "On the levy-walk nature of human mobility," IEEE/ACM Trans. Networking, vol. 19, no. 3, pp. 630-643, Jun. 2011.

[153] Y. Li, et al., "A Markov jump process model for urban vehicular mobility: Modeling and application," IEEE Trans. Mobile Computing, vol. 13, no. 9, pp. 1911-1926, Dec. 2014.

[154] Y. Li, et al., "Limits of predictability for large-scale urban vehicular mobility," IEEE Trans. Intelligent Transport Systems, vol. 15, no. 6 , pp. 2671-2682, Dec. 2014

[155] X. Hou, et al., "Vehicular fog computing: A viewpoint of vehicles as the infrastructures," IEEE Trans. Veh. Techno., vol. 65, no. 6, pp. 38603873, Jun. 2016.

[156] C. Wang, Y. Li, D. Jin, and S. Chen, "On the serviceability of mobile vehicular cloudlets in large-scale urban environment," IEEE Trans. Intelligent Transportation Systems, vol. 17, no. 10, pp. 2960-2970, Oct. 2016.

[157] M. Großmann, "Proximity enhanced mobile D2D video streaming," in Proc. MobiCom 2013 (Istanbul, Turkey), Aug. 22-26, pp. 427-429.

[158] L. Chen, et al., "Mobile relay in LTE-advanced systems," IEEE Commun. Mag., vol. 51, no. 11, pp. 144-151, Nov. 2013

[159] K. Vanganuru, S. Ferrante, and G. Sternberg, "System capacity and coverage of a cellular network with D2D mobile relays," in Proc. MILCOM 2012 (Orlando, USA), Oct. 29-Nov. 1, 2012, pp. 1-6.

[160] C. Gao, Y. Li, and D. Jin, "Mobility assisted device-to-device communications underlaying cellular networks," in Proc. ICNC 2016 (Kauai, USA), Feb. 15-18, 2016, pp. 1-6.
[161] M. Botsov, M. Klügel, W. Kellerer, and P. Fertl, "Location-based resource allocation for mobile D2D communications in multicell deployments," in Proc. ICC 2015 Workshops (London, UK), Jun. 8-12, 2015, pp. 2444-2450.

[162] A. Aijaz, H. Aghvami, and M. Amani, "A survey on mobile data offloading: Technical and business perspectives," IEEE Wireless Commun., vol. 20, no. 2, pp. 104-112, Apr. 2013.

[163] A. Shabani, S. P. Shariatpanahi, V. Shah-Mansouri, and A. Khonsari, "Mobility increases throughput of wireless device-to-device networks with coded caching," in Proc. ICC 2016 ( Kuala Lumpur, Malaysia), May 23-27, 2016, pp. 1-6.

[164] Y. Zhang, et al., "Social network enhanced device-to-device communication underlaying cellular networks," in Proc. ICCC 2013 Workshops (Xian China), Aug. 12-14, 2013, pp. 182-186.

[165] X. Wang, X. Li, and V. C. M. Leung, "TASA: Traffic offloading by tagassisted social-aware opportunistic sharing in mobile social networks," in Proc. 21st Int. Workshop Local and Metropolitan Area Networks (Beijing, China), Apr. 22-24, 2015, pp. 1-6.

[166] X. Wang, Z. Sheng, S. Yang, and V. C. M. Leung, "Tag-assisted socialaware opportunistic device-to-device sharing for traffic offloading in mobile social networks," IEEE Wireless Commun., vol. 23, no. 4, pp. 60 67, Aug. 2016.

[167] L. Pu, X. Chen, J. Xu, and X. Fu, "Content retrieval at the edge: A social-aware and named data cooperative framework," IEEE Trans. Emerging Topics Computing, early access.

[168] J. Rao, H. Feng, and Z. Chen, "Exploiting user mobility for D2D assisted wireless caching networks," in Proc. WCSP 2016 (Yangzhou, China), Oct. 13-15, 2016, pp. 1-5.

[169] M. Chen, et al., "Mobility-aware caching and computation offloading in 5G ultra-dense cellular networks," Sensors, vol. 16, no. 7, pp. 1-13, 2016.

[170] I. Pappalardo, G. Quer, B. D. Rao, and M. Zorzi, "Caching strategies in heterogeneous networks with D2D, small bs and macro bs communications," in 2016 IEEE International Conference on Communications (ICC), May 2016, pp. 1-6.

[171] C. Jarray and A. Giovanidis, "The effects of mobility on the hit performance of cached D2D networks," in 2016 14th International Symposium on Modeling and Optimization in Mobile, Ad Hoc, and Wireless Networks (WiOpt), May 2016, pp. 1-8.

[172] G. Bigwood, et al., "Exploiting self-reported social networks for routing in ubiquitous computing environments," in Proc. WiMob 2008 (Avignon, France), Oct. 12-14, 2008, pp. 484-489.

[173] H. Huang, et al., "Performance evaluation of SUVnet with real-time traffic data," IEEE Trans. Veh. Techno., vol. 56, no. 6, pp. 3381-3396, Nov. 2007.

[174] S. Krishnan and H. S. Dhillon, "Effect of user mobility on the performance of device-to-device networks with distributed caching," IEEE Wireless Comm. Letters, vol. 6, no. 2, pp. 194-197, Apr., 2017.

[175] Wu et al. "The role of mobility for D2D communications in LTEadvanced networks: energy vs. bandwidth efficiency," IEEE Wireless Comm., vol. 21, no. 2, pp. 66-71, Apr., 2014

[176] A. Memon, W. Liu, and A. Al-Anbuky, "Catchme if you can: Enable sustainable communications using internet of movable things," in 14th IEEE Intl. Conf. on Dependable, Autonomic and Secure Computing, pp. 947-952, Aug., 2014.

[177] B. Zhang, Y. Li, D. Jin, and P. Hui, "Adaptive wakeup scheduling based on power-law distributed contacts in delay tolerant networks," in IEEE International Conference on Comm., pp. 409-414, Jun., 2014.

[178] B. B. Bista and D. B. Rawat, "Energy consumption and performance of delay tolerant network routing protocols under different mobility models," in 7th International Conference on Intelligent Systems, Modeling and Simulation, pp. 325-330, Jan., 2016.

[179] A. Vazintari and P. G. Cottis, "Mobility management in energy constrained self-organizing delay tolerant networks: An autonomic scheme based on game theory," IEEE Trans. on Mobile Computing, vol. 15, no. 6, pp. 1401-1411, Jun., 2016.

[180] Y. Cai, D. Wu, and L. Zhou, "Green resource sharing for mobile device-to-device communications," in IEEE Wireless Communication and Networking Conference (WCNC), pp. 3142-3147, Apr., 2014.

[181] A. Moraleda-Soler, B. Coll-Perales, and J. Gozalvez, "Link-aware opportunistic D2D communications: Open source test-bed and experimental insights into their energy, capacity and qos benefits," in 11th International Symposium on Wireless Communications Systems (ISWCS), pp. 606-610, Aug., 2014,

[182] Y. Li, L. Su, D. Jin, and L. Zeng, "Energy-constrained mobility control for epidemic routing in disruption tolerant networks," in Global Telecommunications Conference (GLOBECOM), Jan., 2012. 
[183] O. N. C. Yilmaz, Z. Li, K. Valkealahti, M. A. Uusitalo, M. Moisio, P. Lundén, and C. Wijting, "Smart mobility management for D2D communications in $5 \mathrm{~g}$ networks," in IEEE Wireless Communications and Networking Conference Workshops, pp. 219-223, Apr., 2014.

[184] H. Y. Chen, M. J. Shih, and H. Y. Wei, "Handover mechanism for device-to-device communication," in IEEE Conference on Standards for Communications and Networking, pp. 72-77, Oct., 2015.

[185] S. Yang, U. Adeel, Y. Tahir, and J. A. McCann, "Practical opportunistic data collection in wireless sensor networks with mobile sinks," IEEE Trans. on Mobile Computing, vol. 16, no. 5, pp. 1420-1433, May, 2017.

[186] Y. Li, Z. Wang, L. Su, D. Jin, and S. Chen, "An optimal relaying scheme for delay-tolerant networks with heterogeneous mobile nodes," IEEE Trans. on Vehicular Tech., vol. 62, no. 5, pp. 2239-2252, Jun., 2013.

[187] M. H. Zayani, V. Gauthier, I. Slama, and D. Zeghlache, "Tracking topology dynamicity for link prediction in intermittently connected wireless networks," in 8th International Wireless Communications and Mobile Computing Conference, pp. 469-474, Aug., 2012.

[188] S. Barua and R. Braun, "A novel approach of mobility management for the D2D communications in 5G mobile cellular network system," in 18th APNOMS, pp. 1-4, Oct., 2016.

[189] Y. Li, Z. Wang, D. Jin, L. Su, L. Zeng, and S. Chen, "Optimal relaying in heterogeneous delay tolerant networks," in IEEE International Conference on Commun., pp. 1-5, Jun., 2011.

[190] Z. Wang, L. Sun, M. Zhang, H. Pang, E. Tian, and W. Zhu, "Propagation and mobility-aware D2D social content replication," IEEE Trans. on Mobile Computing, vol. 16, no. 4, pp. 1107-1120, Apr., 2017.

[191] Y. Li, G. Su, D. O. Wu, D. Jin, L. Su, and L. Zeng, "The impact of node selfishness on multicasting in delay tolerant networks," IEEE Trans. on Vehicular Tech., vol. 60, no. 5, pp. 2224-2238, May, 2011.

[192] A. Picu, T. Spyropoulos, and T. Hossmann, "An analysis of the info. spreading delay in heterogeneous mobility DTNs," in World of Wireless, Mob. and Multimedia Net. (WoWMoM), pp. 1-10, Jun., 2012.

[193] Baron et al., "Centrally-controlled mass data offloading using vehicular traffic," IEEE Trans. on Net. and Service Mangt., vol. 14, no., 2, pp. 401415, Jun., 2017.

[194] Y. Li, D. Jin, P. Hui, and S. Chen, "Contact-aware data replication in roadside unit aided vehicular delay tolerant networks," IEEE Trans. on Mobile Computing, vol. 15, no. 2, pp. 306-321, Feb., 2016.

[195] M. Karaliopoulos, "Engage others or leave it to the source? On optimal message replication in DTNs under imperfect cooperation," IEEE Trans. on Mobile Computing, vol. 16, no. 3, pp. 730-743, Mar., 2017.

[196] M. Waqas, M. Zeng, and Y. Li, "Mobility-assisted device to device communications for content transmission," in 13th International Wireless Communications and Mobile Computing Conference (IWCMC), pp. 206-211, Jun., 2017.

[197] M. Waqas, M. Zeng, Y. Li, D. Jin and Z. Han, "Mobility Assisted Content Transmission For Device-to-Device Communication Underlaying Cellular Networks," in IEEE Trans. on Vehicular Tech., vol. 67, no. 7, pp. 6410-6423, Jul., 2018.

[198] Kang et al."Mobile caching policies for device-to-device (D2D) content delivery networking," in IEEE Conference on Computer Communications Workshops, pp. 299-304, Apr., 2014.

[199] H. J. Kang and C. G. Kang, "Mobile device-to-device (D2D) content delivery networking: A design and optimization framework," Journal of Communications and Networks, vol. 16, no. 5, pp. 568-577, Oct., 2014.

[200] K. Henmi and A. Koyama, "A DTN routing protocol based on mobility and maximum number of replications," in 7th Int. Conf. on Complex, Intelligent, and Software Intensive Sys., pp. 293-298, Jul., 2013.

[201] C. Giannini, P. Calegari, C. Buratti, and R. Verdone, "Delay tolerant network for smart city: Exploiting bus mobility," in AEIT International Annual Conference, pp. 1-6, Apr., 2016.

[202] "IEEE stand. for local and metropolitan area networks-part 21.1: Media independent services," IEEE STD. 802.21.1-2017, pp. 1-211, 2017.

[203] S. Andreev, A. Pyattaev, K. Johnsson, O. Galinina, and Y. Koucheryavy, "Cellular traffic offloading onto network-assisted device-to-device connections," IEEE Comm. Magazine, vol. 52, no. 4, pp. 20-31, Apr., 2014.

[204] S. Andreev, O. Galinina, A. Pyattaev, K. Johnsson, and Y. Koucheryavy, "Analyzing assisted offloading of cellular user sessions onto D2D links in unlicensed bands," IEEE Journal on Selected Areas in Communications, vol. 33, no. 1, pp. 67-80, Jan., 2015.

[205] Orsino et al. "Direct connection on the move: Characterization of user mobility in cellular-assisted D2D systems," IEEE Vehicular Technology Magazine, vol. 11, no. 3, pp. 38-48, Sept., 2016.

[206] R. Wang, X. Peng, J. Zhang, and K. B. Letaief, "Mobility-aware caching for content-centric wireless networks: modeling and methodology," IEEE Comm. Mag., vol. 54, no. 8, pp. 77-83, Aug., 2016.
[207] Diggavi et al. "Even one-dimensional mobility increases the capacity of wireless networks," IEEE Trans. on Information Theory, vol. 51, no. 11, pp. 3947-3954, Nov., 2005.

[208] Song et al. "Limits of predictability in human mobility," Science, vol. 327, no. 5968, pp. 1018-1021, 2010.

[209] X. Ge, J. Ye, Y. Yang, and Q. Li, "User mobility evaluation for 5G small cell networks based on individual mobility model," IEEE Journal on Selected Areas in Comm., vol. 34, no. 3, pp. 528-541, Mar., 2016.

[210] I. R. N. M. Shin, S. H. N. K. Lee, and S. Chong, "Human mobility patterns and their impact on routing in human-driven mobile networks," in HotNets-VI, 2007.

[211] M. Kim, D. Kotz, and S. Kim, "Extracting a mobility model from real user traces," in 25th IEEE International Conference on Computer Communications, pp. 1-13, Apr., 2006.

[212] R. Beuran, S. Miwa, and Y. Shinoda, "Behavioral mobility model with geographic constraints," in 27th Int. Conf. on Advanced Information Networking and Applications Workshops, pp. 470-477, Mar., 2013.

[213] Y. Cao, C. Long, T. Jiang, and S. Mao, "Share communication and computation resources on mobile devices: a social awareness perspective," IEEE Wireless Comm., vol. 23, no. 4, pp. 52-59, Aug., 2016.

[214] T. Maksymyuk, M. Brych, and V. Pelishok, "Stochastic geometry models for 5G heterogeneous mobile networks." Smart CR, vol. 5, no. 2, pp. 89-101, 2015.

[215] C. Boldrini and A. Passarella, "HCMM: Modelling spatial and temporal properties of human mobility driven by users' social relationships," Computer Communications, vol. 33, no. 9, pp. 1056-1074, 2010.

[216] M. Chen, Y. Hao, Y. Li, C. F. Lai, and D. Wu, "On the computation offloading at ad hoc cloudlet: architecture and service modes," IEEE Communications Magazine, vol. 53, no. 6, pp. 18-24, Jun., 2015.

[217] Z. Su, Y. Hui, and S. Guo, "D2D-based content delivery with parked vehicles in vehicular social networks," IEEE Wireless Communications, vol. 23, no. 4, pp. 90-95, Aug., 2016.

[218] Jo et al. "Device-to-device-based heterogeneous radio access network architecture for mobile cloud computing," IEEE Wireless Comm., vol. 22, no. 3, pp. 50-58, Jun., 2015

[219] M. E. Newman, "The structure and function of complex networks," SIAM review, vol. 45, no. 2, pp. 167-256, 2003.

[220] A.-L. Barabási and R. Albert, "Emergence of scaling in random networks," science, vol. 286, no. 5439, pp. 509-512, 1999.

[221] L. C. Freeman, "Centrality in social networks conceptual clarification," Social networks, vol. 1, no. 3, pp. 215-239, 1978.

[222] Ai et al. "Future railway services-oriented mobile communications network," IEEE Comm. Mag., vol. 53, no. 10, pp. 78-85, Oct., 2015.

[223] W. J. Martin, The global information society. Routledge, 2017.

[224] Park et al. "Integrated traffic-communication simulation evaluation environment for intel drive applications using SAE j2735 message sets," Transportation Research Record: Journal of the Transportation Research Board, no. 2243, pp. 117-126, 2011.

[225] M. Piórkowski, N. Sarafijanovic-Djukic, and M. Grossglauser, "On clustering phenomenon in mobile partitioned networks," in 1st ACM SIGMOBILE workshop on Mobility models. pp. 1-8, 2008.

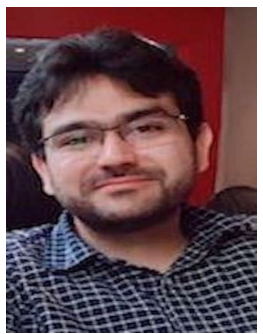

Muhammad Waqas received the B.Sc. and M.Sc. degrees from the Department of Electrical Engineering, University of Engineering and Technology Peshawar, Pakistan, in 2009 and 2014, respectively. From 2012 to 2015, he has also served Sarhad University of Science and Information Technology, Peshawar, Pakistan, as an assistant professor and program coordinator.

$\mathrm{He}$ is currently pursuing his Ph.D. degree with the Beijing National Research Center for Information Science and Technology, Department of Electronic Engineering, Tsinghua University, Beijing, China. He has several research publications in IEEE Journals and Conferences. His current research interests are in the areas of networking and communications including $5 \mathrm{G}$ networks, D2D communication resource allocation and physical layer security and information security, mobility investigation in D2D communication, Fog computing and MEC. 


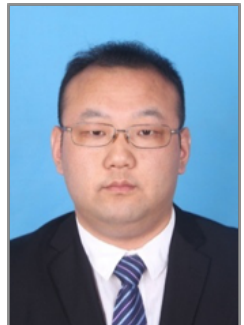

Yong Niu (M'17) received the B.E. degree in Electrical Engineering from Beijing Jiaotong University, China, in 2011, and the Ph.D. degree in Electronic Engineering from Tsinghua University, Beijing, China, in 2016. From 2014 to 2015, he was a Visiting Scholar with the University of Florida, Gainesville, FL, USA. He is currently an Associate Professor with the State Key Laboratory of Rail Traffic Control and Safety, Beijing Jiaotong University. His research interests are in the areas of networking and communications, including millimeter wave communications, device-to-device communication, medium access control, and software-defined networks. He received the Ph.D. National Scholarship of China in 2015, the Outstanding Ph.D. Graduates and Outstanding Doctoral Thesis of Tsinghua University in 2016, the Outstanding Ph.D. Graduates of Beijing in 2016, and the Outstanding Doctorate Dissertation Award from the Chinese Institute of Electronics in 2017. He has served as Technical Program Committee member for IWCMC 2017, VTC2018-Spring, IWCMC 2018, INFOCOM 2018, and ICC 2018. He was the Session Chair for IWCMC 2017. He was the recipient of the 2018 International Union of Radio Science Young Scientist Award.

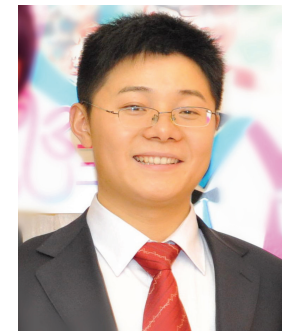

Yong Li (M'09-SM'16) received the B.S. degree in electronics and information engineering from Huazhong University of Science and Technology, Wuhan, China, in 2007 and the Ph.D. degree in electronic engineering from Tsinghua University, Beijing, China, in 2012. He is currently a Faculty Member of the Department of Electronic Engineering, Tsinghua University.

Dr. Li has served as General Chair, TPC Chair, TPC Member for several international workshops and conferences, and he is on the editorial board of three international journals. His papers have total citations more than 2300 (six papers exceed 100 citations, Google Scholar). Among them, eight are ESI Highly Cited Papers in Computer Science, and four receive conference Best Paper (run-up) Awards. He received IEEE 2016 ComSoc Asia-Pacific Outstanding Young Researchers and Young Talent Program of China Association for Science and Technology.

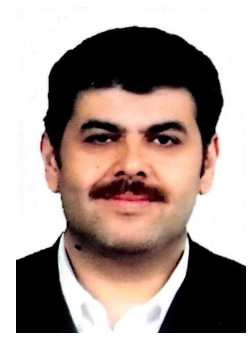

Manzoor Ahmed received the B.E. and M.S. degrees in electrical engineering and computer science from Balochistan Engineering University, Khuzdar, Pakistan, in 1996 and 2010, respectively, and the Ph.D. degree in communication and information systems from the Beijing University of Posts and Telecommunications, China, in 2015. From 1997 to 2000, he was a Lecturer with Balochistan Engineering University and a Telecomm Engineer in government-owned telecommunication service provider NTC, Pakistan, from 2000 to 2011 . He was a Postdoctoral Researcher from the Electrical Engineering Department, Tsinghua University, China, from 2015 to 2018. He is currently a Faculty Member with the Department of Computer Science and Technology, Qingdao University. His research interests include resource allocation and offloading in vehicular communications and networking, fog and edge computing, socially aware D2D communication, and physical layer security. He has several research publications in IEEE top journals and conferences. He received several awards, including the Distinction Award from the President of Pakistan, the Best Employee Award from NTC, and the Best Paper Award from the 2014 GameNets Conference.

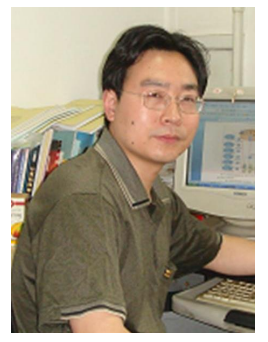

Depeng Jin (M'2009) received his B.S., M.S. and $\mathrm{Ph} . \mathrm{D}$. degrees from Tsinghua University in 1995 , 1997 and 2000, respectively. From 2000 to 2003, he was an assistant professor. From 2004 to 2012, he was an associate professor. Since 2012, he has been a Professor with Department of Electronic Engineering, Tsinghua University. His research focuses on communication and networking. His current interests are in the topics of software-defined networks, wireless mobile network, and mobile big data.

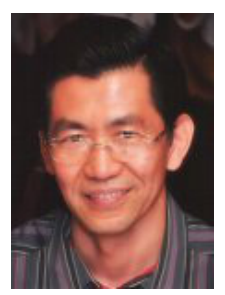

Sheng Chen (M'90-SM'97-F'08) received his BEng degree from the East China Petroleum Institute, Dongying, China, in 1982, and his $\mathrm{PhD}$ degree from the City University, London, in 1986, both in control engineering. In 2005, he was awarded the higher doctoral degree, Doctor of Sciences (DSc), from the University of Southampton, Southampton, UK. From 1986 to 1999, He held research and academic appointments at the Universities of Sheffield, Edinburgh and Portsmouth, all in UK. Since 1999, he has been with the School of Electronics and Computer Science, the University of Southampton, UK, where he holds the post of Professor in Intelligent Systems and Signal Processing. Dr Chen's research interests include adaptive signal processing, wireless communications, modelling and identification of nonlinear systems, neural network and machine learning, intelligent control system design, evolutionary computation methods and optimisation. He has published over 650 research papers. Dr. Chen is a Fellow of the United Kingdom Royal Academy of Engineering, a Fellow of IET, a Distinguished Adjunct Professor at King Abdulaziz University, Jeddah, Saudi Arabia, and an original ISI highly cited researcher in engineering (March 2004). Professor Chen has 13,000+ Web of Science citations and $27,000+$ Google Scholar citations.

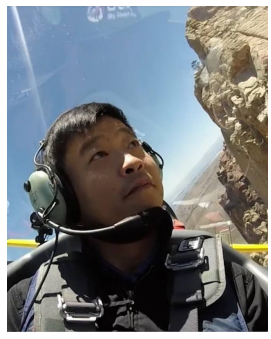

Zhu Han (S'01-M'04-SM'09-F'14) received the B.S. degree in electronic engineering from Tsinghua University, in 1997, and the M.S. and Ph.D. degrees in electrical and computer engineering from the University of Maryland, College Park, in 1999 and 2003, respectively.

From 2000 to 2002, he was an R\&D Engineer of JDSU, Germantown, Maryland. From 2003 to 2006, he was a Research Associate at the University of Maryland. From 2006 to 2008, he was an assistant professor at Boise State University, Idaho. Currently, he is a Professor in the Electrical and Computer Engineering Department as well as in the Computer Science Department at the University of Houston, Texas. His research interests include wireless resource allocation and management, wireless communications and networking, game theory, big data analysis, security, and smart grid. Dr. Han received an NSF Career Award in 2010, the Fred W. Ellersick Prize of the IEEE Communication Society in 2011, the EURASIP Best Paper Award for the Journal on Advances in Signal Processing in 2015, IEEE Leonard G. Abraham Prize in the field of Communications Systems (best paper award in IEEE JSAC) in 2016, and several best paper awards in IEEE conferences. Currently, Dr. Zhu Han is an IEEE Communications Society Distinguished Lecturer. 